\title{
On the Absence of Excited Eigenstates of Atoms in QED
}

\author{
Jürg Fröhlich* \\ Institute of Theoretical Physics; ETH Zürich; \\ CH-8093 Zürich, Switzerland \\ (juerg@itp.phys.ethz.ch) \\ Alessandro Pizzo \\ Institute of Theoretical Physics; ETH Zürich; \\ CH-8093 Zürich, Switzerland \\ (pizzo@itp.phys.ethz.ch) \\ 18-April-2007
}

\begin{abstract}
For the standard model of QED with static nuclei, nonrelativistic electrons and an ultraviolet cutoff, a new simple proof of absence of excited eigenstates with energies above the groundstate energy and below the ionization threshold of an atom is presented. Our proof is based on a multi-scale virial argument and exploits the fact that, in perturbation theory, excited atomic states decay by emission of one or two photons. Our arguments do not require an infrared cutoff (or regularization) and are applicable for all energies above the groundstate energy, except in a small ( $\alpha$-dependent) interval around the ionization threshold.
\end{abstract}

*also at IHES, Bures-sur-Yvette 


\section{Description of the Problem and Summary of Main Results}

Radiative transitions are responsible for the disappearance of eigenvalues corresponding to excited eigenstates from the spectrum of Hamiltonians describing atoms or molecules coupled to the quantized radiation field. The known mathematical proofs of this fact involve steps only indirectly linked to the physical intuition about this phenomenon. In nonrelativistic QED, previous methods used to analyze the spectrum of such Hamiltonians are based either on the method of complex spectral deformations, showing that higher eigenvalues of the unperturbed Hamiltonian migrate to the lower complex half-plane when the electrons are coupled to quantized radiation field, or on a variant of Mourre's method of positive commutators, which can be used to exclude eigenvalues in certain energy intervals. There is a large body of literature on this subject, dealing with different variants of the standard model of nonrelativistic QED; see e.g. [2], [3], [4], [6], [7], [10], [9], [14], [18]. For a review of previous work, we also refer the reader to the Introduction of [9]. General references on the method of complex spectral deformations and on Mourre's theory are [16], [17], [11].

We begin by summarizing some earlier results closely related to those proven in the present paper.

1) Outside some $\mathcal{O}\left(g^{2}\right)$-neighborhoods (where $g$ is a coupling constant) of the groundstate energy and of the ionization threshold, proofs for the absence of point spectrum and absolute continuity of the energy spectrum have been given in [2] in the presence of an infrared regularization of the form factor in the interaction coupling the electrons to the quantized radiation field. These proofs are based on an operator renormalization group analysis of the spectrum of a dilated Hamiltonian.

2) An approach involving positive commutators [4], as well as a refined version [3] of the complex spectral deformation method yield analogous results, but without any infrared regularization.

The results described in 1) and 2), however, apply only to situations where the decay of an excited state takes place as a consequence of a dipole transition. Hence the assumptions in [2], [3], and [4] do, in general, not cover the entire interval between the groundstate energy and the ionization threshold. For example, the decay of the 2 s level of the hydrogen atom, which is due to two-photon or multi-photon transitions, is not understood in these references.

3) Absence of eigenvalues and absolute continuity of the energy spectrum in a neighborhood of the groundstate energy has recently been proven in [9] 
without any infrared regularization, using a multi-scale version of Mourre's theory.

The purpose of this paper is twofold. First, we prove a complete result for the absence of point spectrum below the (unperturbed) ionization threshold and above the groundstate energy, at least for the hydrogen atom. Moreover, and more importantly, we present a proof closely related to the usual physical arguments of perturbation theory, which are made mathematically precise in this paper. Our starting point is the spectroscopic evidence that one- and two-photon transitions are responsible for the decay of all excited atomic or molecular bound states. In fact, starting from a perturbative expansion for a putative excited eigenstate of the total Hamiltonian, we arrive at a contradiction by taking scalar products of the putative eigenvector with trial vectors. These trial vectors represent the decay products of the putative excited states after emission of one or two photons. The perturbative nature of any solution of the eigenvalue equation, should one exist, is derived from a multiscale virial argument. Our multiscale virial argument yields an estimate of the expected number of photons in a putative excited eigenstate of energy below the ionization threshold. An outline of our main arguments is presented at the end of this section.

Definition of the model

In the standard model of QED with nonrelativistic matter, an atom or molecule is described as a quantum-mechanical bound state consisting of static, positively charged, pointlike nuclei surrounded by pointlike electrons of charge $-e$ and spin $\frac{1}{2}$ with nonrelativistic kinematics, (Pauli electrons).

For simplicity, the system studied in this paper is a hydrogen atom consisting of a single, static proton and one electron. The electron is bound to the proton by electrostatic Coulomb attraction, and it interacts with the transverse soft modes of the quantized electromagnetic field. We eliminate ultraviolet divergences by imposing an ultraviolet cutoff on the interactions between the electron and the photons. For simplicity of presentation, we neglect the spin of the electron, so that the Zeeman coupling of the electron's magnetic moment to the quantized magnetic field is turned off.

In the following, we choose the same units as in [1]: The electron position is measured in units of $\frac{1}{2} r_{B o h r}$, and one unit of energy corresponds to 4 Rydberg.

The Hilbert space of pure state vectors of the system is given by

$$
\mathcal{H}:=\mathcal{F}_{\text {phys. }} \otimes \mathcal{H}_{\text {el }}
$$

where $\mathcal{H}_{e l}=L^{2}\left(\mathbb{R}^{3}\right)$ is the Hilbert space appropriate to describe states of a single electron (neglecting its spin), and $\mathcal{F}_{\text {phys }}$. is the Fock space used to describe the states of the transverse modes of the quantized electromagnetic 
field, i.e., the photons. This space is defined starting from the Fock space $\mathcal{F}$ of general vector bosons, which is given by

$$
\mathcal{F}:=\bigoplus_{N=0}^{\infty} \mathcal{F}^{(N)}, \quad \mathcal{F}^{(0)}=\mathbb{C} \Omega
$$

where $\Omega$ is the vacuum vector, i.e., the state without any vector boson, and the state space, $\mathcal{F}^{(N)}$, of $N$ vector bosons is given by

$$
\mathcal{F}^{(N)}:=\mathcal{S}_{N} \mathfrak{h}^{\otimes N}, \quad N \geq 1,
$$

where the Hilbert space, $\mathfrak{h}$, of state vectors of a single vector boson is given by

$$
\mathfrak{h}:=L^{2}\left[\mathbb{R}^{3} \otimes \mathbb{C}^{3}\right] .
$$

In (I.4), $\mathbb{R}^{3}$ is the momentum space of our vector bosons, and $\mathbb{C}^{3}$ accounts for the three independent vector components. In Eq. (I.3), $\mathcal{S}_{N}$ denotes the orthogonal projection onto the subspace of $\mathfrak{h}^{\otimes N}$ of totally symmetric $N$ particle wave functions.

The physical Fock space, $\mathcal{F}_{\text {phys. }}$, which describes the states of photons is the subspace contained in $\mathcal{F}$ spanned by wave functions

$$
\frac{1}{N !} \sum_{\mathcal{P}_{N}}\left[\vec{f}_{p_{1}}\left(\vec{k}_{1}\right)\right] \otimes \cdots \otimes\left[\vec{f}_{p_{N}}\left(\vec{k}_{N}\right)\right] \in \mathcal{S}_{N} \mathfrak{h}^{\otimes N}
$$

where $\mathcal{P}_{N}$ is the permutation group of $N$-elements, and $\left[\overrightarrow{f_{l}}(\vec{k})\right]$ is a column vector with components $f_{l}^{(1)}(\vec{k}), f_{l}^{(2)}(\vec{k}), f_{l}^{(3)}(\vec{k})$, which is transverse, i.e.,

$$
\sum_{j=1,2,3} k_{j} f_{l}^{(j)}(\vec{k})=0, \quad \vec{k}=\left(k_{1}, k_{2}, k_{3}\right) .
$$

The dynamics of the system is generated by the Hamiltonian

$$
H_{\alpha}:=\left(-i \vec{\nabla}_{\vec{x}}+\alpha^{3 / 2} \vec{A}(\alpha \vec{x})\right)^{2}-\frac{1}{|\vec{x}|}+H^{f} .
$$

Here, $\vec{\nabla}_{\vec{x}}$ denotes the gradient with respect to the electron position variable $\vec{x} \in \mathbb{R}^{3}, \alpha \cong 1 / 137$ is the feinstructure constant, $\vec{A}(\vec{x})$ denotes the vector potential of the transverse modes of the quantized electromagnetic field in the Coulomb gauge,

$$
\vec{\nabla}_{\vec{x}} \cdot \vec{A}(\vec{x})=0
$$

with an ultraviolet cutoff imposed on the high-frequency modes, and $-\frac{1}{|\vec{x}|}$ is the Coulomb potential of electrostatic attraction of the electron to the nucleus. The atomic Hamiltonian, $H_{e l}$, is defined by

$$
H_{e l}:=-\Delta_{\vec{x}}-\frac{1}{|\vec{x}|},
$$


where $\Delta_{\vec{x}}$ is the Laplacian.

In equation (I.5), $H^{f}$ is the Hamiltonian of quantized, free massless vector bosons. It is given by

$$
H^{f}:=\sum_{i=1,2,3} \int d^{3} k a_{i}^{*}(\vec{k})|\vec{k}| a_{i}(\vec{k})
$$

where $a_{i}^{*}(\vec{k})$ and $a_{i}(\vec{k})$ are the usual creation- and annihilation operators obeying the canonical commutation relations

$$
\begin{gathered}
{\left[a_{i}^{*}(\vec{k}), a_{i^{\prime}}^{*}\left(\vec{k}^{\prime}\right)\right]=\left[a_{i}(\vec{k}), a_{i^{\prime}}\left(\vec{k}^{\prime}\right)\right]=0,} \\
{\left[a_{i}(\vec{k}), a_{i^{\prime}}^{*}\left(\vec{k}^{\prime}\right)\right]=\delta_{i, i^{\prime}} \delta\left(\vec{k}-\vec{k}^{\prime}\right)} \\
a_{i}(\vec{k}) \Omega=0
\end{gathered}
$$

for all $\vec{k}, \vec{k}^{\prime} \in \mathbb{R}^{3}$ and $i, i^{\prime}=1,2,3$.

The vector potential in the Coulomb gauge is given by

$$
\begin{aligned}
& A_{j}(\vec{x}):= \\
& \quad \frac{1}{(2 \pi)^{3 / 2}} \sum_{l=1,2,3} \int \frac{d^{3} k}{\sqrt{2|\vec{k}|}} \Lambda(\vec{k})\left\{e^{-i \vec{k} \cdot \vec{x}} P_{j, l}^{\perp}(\vec{k}) a_{l}^{*}(\vec{k})+e^{i \vec{k} \cdot \vec{x}} P_{j, l}^{\perp}(\vec{k}) a_{l}(\vec{k})\right\}
\end{aligned}
$$

where $\Lambda(\vec{k})$ is a nonnegative, smooth approximation of the characteristic function of the ball $\left\{\vec{k} \in \mathbb{R}^{3}|| \vec{k} \mid \leq \kappa\right\}$, and

$$
P_{j, l}^{\perp}(\vec{k}):=\delta_{j, l}-\frac{k_{j} k_{l}}{|\vec{k}|^{2}}
$$

The equation

$$
k_{j} P_{j, l}^{\perp}(\vec{k})=k_{j} \delta_{j, l}-k_{j} \frac{k_{j} k_{l}}{|\vec{k}|^{2}}=0,
$$

where, here and in the following, repeated indices are summed over, expresses the Coulomb gauge condition.

The cut-off function $\Lambda(\vec{k})$ ensures that modes of the electromagnetic field corresponding to wave vectors $\vec{k}$ with $|\vec{k}| \geq \kappa$ do not interact with the electron, (ultraviolet cutoff); $\kappa$ can be chosen to correspond roughly to the rest energy of an electron, and, throughout our analysis, will be kept fixed and larger than the absolute value of the groundstate energy of the unperturbed system. 
Given an operator-valued function $F: \mathbb{R}^{3} \times J \rightarrow \mathcal{B}\left(\mathcal{H}_{e l}\right), J:=\{1,2,3\}$, with $k_{j} F(\vec{k}, j)=0$, we write

$$
\begin{aligned}
a^{*}(F) & :=\sum_{j=1,2,3} \int a_{j}^{*}(\vec{k}) \otimes F(\vec{k}, j) d^{3} k, \\
a(F) & :=\sum_{j=1,2,3} \int a_{j}(\vec{k}) \otimes F(\vec{k}, j)^{*} d^{3} k .
\end{aligned}
$$

This allows us to write the velocity operator $\vec{v}$ (rescaled by 2) as

$$
\vec{v} \equiv \vec{v}^{\alpha}:=-i \vec{\nabla}_{x}+a^{*}\left(\vec{G}_{\vec{x}}^{\alpha}\right)+a\left(\vec{G}_{\vec{x}}^{\alpha}\right),
$$

where $\vec{G}_{\vec{x}}^{\alpha}: \mathbb{R}^{3} \times J \rightarrow \mathcal{B}\left(\mathcal{H}_{e l}\right)^{3}$ are the multiplication operators defined by

$$
G_{j, \vec{x}}^{\alpha}(\vec{k}, l):=\frac{\alpha^{3 / 2}}{(2 \pi)^{3 / 2}} \frac{\Lambda(\vec{k})}{\sqrt{2|\vec{k}|}} e^{-i \alpha \vec{k} \cdot \vec{x}} P_{j, l}^{\perp}(\vec{k}) .
$$

In terms of the velocity operator, the Hamiltonian has the simple form

$$
H_{\alpha}=\left(\vec{v}^{\alpha}\right)^{2}-\frac{1}{|\vec{x}|}+H^{f} .
$$

We proceed to recall some basic properties of the Hamiltonian $H_{\alpha}$ and of other operators that will be used in the following sections.

For sufficiently small values of $\alpha$, the Hamiltonian $H_{\alpha}$ is selfadjoint on its domain $\mathcal{D}\left(H_{\alpha}\right)=\mathcal{D}\left(H_{0}\right)$, where $\mathcal{D}\left(H_{0}\right)$ is the domain of the selfadjoint operator

$$
H_{0}:=-\Delta_{\vec{x}}-\frac{1}{|\vec{x}|}+H^{f} .
$$

The operator $H_{\alpha}$ is bounded from below, and the infimum of the spectrum is a non-degenerate eigenvalue, the groundstate energy, $E_{g s} \equiv E_{g s}(\alpha)$, corresponding to a unique eigenvector, $\psi_{g s}$; see, e.g., [1],[3] ( $\alpha$ small), and [13] ( $\alpha$ arbitrary).

The atomic Hamiltonian

$$
H_{e l}:=-\Delta_{\vec{x}}-\frac{1}{|\vec{x}|}
$$

has an ionization threshold $\Sigma=0$, above which the spectrum is absolutely continuous, and the electron is not bound to the nucleus, anymore. By $\mathcal{H}_{e l}^{(j)}$, $j \geq 1$, we denote the subspace of eigenstates of $H_{e l}$ corresponding to the $j^{t h}$ excited energy level; $\mathcal{H}_{e l}^{(0)}$ is the one-dimensional subspace $\left\{\mathbb{C} \phi^{(0)}\right\}$ of $\mathcal{H}_{e l}$, where $\phi^{(0)}$ is the unique groundstate of $H_{e l}$. We recall that the degeneracy of $\mathcal{H}_{e l}^{(j)}$ is $(j+1)^{2}$.

Some crucial properties of the eigenfunctions of $H_{e l}$ are summarized below. 
(P1) Given any state $\phi^{(j)} \in \mathcal{H}_{\text {el }}^{(j)}$, with $j \geq 2$, or with $j=1$ and for an angular momentum of $\phi^{(1)}$ different from zero, there is a state $\phi^{(i)} \in$ $\mathcal{H}_{\text {el }}^{(i)}$, for some $i$ with $i<j$, such that

$$
\left|\left(\phi^{(i)}, \vec{p} \phi^{(j)}\right)\right|>0
$$

where $\vec{p}=-i \vec{\nabla} \vec{x}$.

(P2) The scalar product below is different from zero:

$$
\left(\phi^{(0)}, p_{1} P_{\mathcal{H}_{e l}^{(1)}} x_{1} \phi_{l=0}^{(1)}\right),
$$

where $P_{\mathcal{H}_{e l}^{(1)}}$ is the spectral projection on the subspace $\mathcal{H}_{\text {el }}^{(1)}$, and $\phi_{l=0}^{(1)}$ has angular momentum equal to 0.

Property (P1) is typically true, i.e., up to miraculous selection rules, one can check it either starting from the analytic expression of the radial matrix elements (see [15]), or by using classical methods for the approximate calculation of these matrix elements (see [5]), in different regimes depending on the energy levels, $E^{(i)}, E^{(j)}$, of the two considered states, on the size of their difference, $E^{(j)}-E^{(i)}$, and on the angular momenta. In this paper, we assume Property (P1) to be always fulfilled. Property (P2) can be checked by an elementary calculation.

Notation

1) The symbol $\|$.$\| denotes the norm of vectors in \mathcal{H}$ and of operators on $\mathcal{H}$. The symbol $\|\cdot\|_{\mathfrak{h}}$ is used for the vector and the operator norm on $\mathfrak{h}$ and $\mathcal{B}(\mathfrak{h})$, respectively.

2) Given a selfadjoint operator, $b$, on the one-particle subspace of Fock space $\mathcal{F}, d \Gamma(b)$ denotes the corresponding second-quantized operator acting on $\mathcal{F}$.

3) Throughout our paper, the symbol $\mathcal{O}\left(\alpha^{n}\right)$ stands for a positive quantity bounded from above by const $\cdot \alpha^{n}$, where the constant is independent of $\alpha$, unless specified otherwise.

Summary of key ideas of proof

The main result of our paper is stated in the following

\section{Theorem}

For arbitrary $\Delta_{\Sigma}>0$, there is a constant $\bar{\alpha}\left(\Delta_{\Sigma}\right)>0$ such that, for $|\alpha|<\bar{\alpha}\left(\Delta_{\Sigma}\right)$, the Hamiltonian $H_{\alpha}$ does not have any eigenvalue in the energy interval $\left(E_{g s}(\alpha), \Sigma-\Delta_{\Sigma}\right)$. 
The proof of this result (see Corollary III.3) is completed in Section III, using a multiscale virial argument presented in Section II.

As mentioned above, our proof of this result is indirect. We assume that an eigenvector $\psi_{\alpha} \in \mathcal{H}$ of $H_{\alpha}$ exists, with

$$
H_{\alpha} \psi_{\alpha}=E_{\alpha} \psi_{\alpha}
$$

for some energy $E_{\alpha} \in\left(E_{g s}, \Sigma\right)$. Then, using a multiscale virial argument (see Theorem II.1.), we conclude that

$$
\left(\psi_{\alpha}, N^{f} \psi_{\alpha}\right) \leq \mathcal{O}\left(\alpha^{3}\right)
$$

where $N^{f}:=\sum_{l=1,2,3} \int d^{3} k a_{l}^{*}(\vec{k}) a_{l}(\vec{k})$ is the number operator. Our multiscale virial argument involves the dilatation operators

$$
d_{n}:=\frac{1}{2} \chi_{n}(|\vec{k}|)\left[\vec{k} \cdot i \vec{\nabla}_{\vec{k}}+i \vec{\nabla}_{\vec{k}} \cdot \vec{k}\right] \chi_{n}(|\vec{k}|)
$$

on the one-particle space $\mathfrak{h}$, (see Section II.1), where $\chi_{n}$ is a suitable smooth approximation to the characteristic function of the interval $\left[\frac{1}{n+1}, \frac{1}{n}\right]$ contained in the positive frequency half axis, $n=1,2, \ldots$, and $\chi_{0}$ is a suitable smooth approximation to the characteristic function of the interval $[1,+\infty)$. In Lemma II.1., the eigenvalue equation (I.24) and the bound (I.25) are combined to conclude that the vector $\psi_{\alpha}$ and the eigenvalue $E_{\alpha}$ must have the following asymptotic expansions in $\alpha$ : For some $j \geq 0$,

$$
\begin{gathered}
\left\|\psi_{\alpha}-\Omega \otimes \phi^{(j)}\right\|^{2} \leq \mathcal{O}\left(\alpha^{3}\right), \\
\left|E_{\alpha}-E^{(j)}\right| \leq \mathcal{O}\left(\alpha^{3}\right) .
\end{gathered}
$$

This result implies that putative eigenvalues of $H_{\alpha}$ lie in $\mathcal{O}\left(\alpha^{3}\right)$-neighborhoods of eigenvalues of the Hamiltonian $H_{0}$.

A neighborhood of the groundstate energy $E^{(0)}$ is trivially excluded, for small $\alpha$, by combining (I.27) with the fact that $\Omega \otimes \phi^{(0)}$ is asymptotic to the unique groundstate, $\psi_{g s}(\alpha)$, of the Hamiltonian $H_{\alpha}$, as $\alpha \rightarrow 0$, i.e.,

$$
\left\|\Omega \otimes \phi^{(0)}-\psi_{g s}(\alpha)\right\|=o(1) ;
$$

see $[1]$.

A more refined argument is necessary to exclude point spectrum in $\mathcal{O}\left(\alpha^{3}\right)$ neighborhoods of the excited eigenvalues of the Hamiltonian $H_{0}$. Heuristically, this argument goes as follows.

A formal asymptotic expansion of $\psi_{\alpha}$ in powers of $\alpha^{\frac{3}{2}}$ yields the equation

$$
\psi_{\alpha}=\Omega \otimes \phi^{(j)}+\frac{2 \alpha^{\frac{3}{2}}}{H_{0}-E^{(j)}} \vec{p} \cdot \vec{A}(0) \Omega \otimes \phi^{(j)}+o\left(\alpha^{\frac{3}{2}}\right) .
$$


This expansion is ill-defined whenever the range of photon frequencies $(0, \kappa)$ is such that, for some $\vec{k}$ with $|\vec{k}| \in(0, \kappa)$ and some $i<j$,

$$
E^{(j)}-E^{(i)}=|\vec{k}|
$$

and Property (P1) is fulfilled, i.e.,

$$
\left|\left(\phi^{(i)}, \vec{p} \phi^{(j)}\right)\right|>0 .
$$

In fact, in this situation the vector $\vec{p} \cdot \vec{A}(0) \Omega \otimes \phi^{(j)}$ is not in the domain of definition of $\left(H_{0}-E^{(j)}\right)^{-1}$, and hence

$$
\frac{1}{H_{0}-E^{(j)}} \vec{p} \cdot \vec{A}(0) \Omega \otimes \phi^{(j)}
$$

is not a vector in the Hilbert space.

One may then expect that the nonexistence of the vector in (I.32) and the asymptotic expansions in (I.27) and (I.28) yield a contradiction. The key idea to substantiate this expectation is to introduce a trial vector in $\mathcal{H}$ of the following type

$$
\eta^{(i)}:=\int d^{3} k \frac{1}{\epsilon^{\frac{1}{2}}} h\left(\frac{|\vec{k}|-E_{\alpha}+E^{(i)}}{\epsilon}\right) \theta_{l}(\hat{k}) a_{l}^{*}(\vec{k}) \Omega \otimes \phi^{(i)},
$$

with spectral support w.r. to $H_{0}$ peaked at the resonance energy $E_{\alpha} \approx E^{(j)}$, for $\epsilon>0$ small enough (see Theorem III.2. for a precise definition), and to consider the scalar product

$$
\begin{aligned}
& \alpha^{\frac{3}{2}}\left(\left(H_{0}-E^{(j)}\right) \eta^{(i)}, \frac{2}{H_{0}-E^{(j)}} \vec{p} \cdot \vec{A}(0) \Omega \otimes \phi^{(j)}\right) \\
= & 2 \alpha^{\frac{3}{2}}\left(\eta^{(i)}, \vec{p} \cdot \vec{A}(0) \Omega \otimes \phi^{(j)}\right),
\end{aligned}
$$

which is well defined.

The expression above vanishes, as $\epsilon \rightarrow 0$, but, thanks to (I.31), and for a suitable choice of the functions $\theta_{l}(\hat{k})$, the following estimate from below holds

$$
2 \alpha^{\frac{3}{2}}\left|\left(\eta^{(i)}, \vec{p} \cdot \vec{A}(0) \Omega \otimes \phi^{(j)}\right)\right|>Q_{1} \alpha^{\frac{3}{2}} \epsilon^{\frac{1}{2}},
$$

where $Q_{1}$ is an $\epsilon$ - and $\alpha$ - independent constant.

Notice however that, in (I.35), the vector

$$
2 \alpha^{\frac{3}{2}} \vec{p} \cdot \vec{A}(0) \Omega \otimes \phi^{(j)}
$$

can be replaced by

$$
-\left(H_{0}-E_{\alpha}\right)\left(\psi_{\alpha}-\Omega \otimes \phi^{(j)}\right)
$$


up to corrections $o\left(\alpha^{\frac{3}{2}}\right)$, because of (I.27) and (I.28). This is made precise in Section III. Hence,

$$
\begin{aligned}
2 & \alpha^{\frac{3}{2}}\left(\eta^{(i)}, \vec{p} \cdot \vec{A}(0) \Omega \otimes \phi^{(j)}\right)= \\
& =-\left(\eta^{(i)},\left(H_{0}-E_{\alpha}\right)\left(\psi_{\alpha}-\Omega \otimes \phi^{(j)}\right)\right)+\mathcal{O}\left(\alpha^{3}\right) \\
& =-\left(\left(H_{0}-E_{\alpha}\right) \eta^{(i)},\left(\psi_{\alpha}-\Omega \otimes \phi^{(j)}\right)\right)+\mathcal{O}\left(\alpha^{3}\right) .
\end{aligned}
$$

We now observe that the norm $\left\|\left(H_{0}-E_{\alpha}\right) \eta^{(i)}\right\|$ is proportional to $\epsilon$, and therefore, using the Schwarz inequality and (I.27), the absolute value of the scalar product (I.39) is bounded from above by const $\alpha^{\frac{3}{2}} \epsilon$ :

$$
\left|\left(\left(H_{0}-E_{\alpha}\right) \eta^{(i)},\left(\psi_{\alpha}-\Omega \otimes \phi^{(j)}\right)\right)\right| \leq \mathcal{O}\left(\alpha^{\frac{3}{2}} \epsilon\right) .
$$

The bounds (I.36),(I.40) and (I.41) imply that

$$
Q_{1} \alpha^{\frac{3}{2}} \epsilon^{\frac{1}{2}} \leq 2 \alpha^{\frac{3}{2}}\left|\left(\eta^{(i)}, \vec{p} \cdot \vec{A}(0) \Omega \otimes \phi^{(j)}\right)\right| \leq \mathcal{O}\left(\alpha^{\frac{3}{2}} \epsilon\right)+\mathcal{O}\left(\alpha^{3}\right),
$$

which yields a contradiction for $\epsilon$ proportional to $\alpha$ and $\alpha$ small enough.

This is the essence of our arguments for dipole transitions. For precise statements see Theorem III.2. For the decay of the state $\phi_{l=0}^{(1)}$ (the first excited atomic state with angular momentum $l=0$ ), we are forced to consider a twophoton transition, and a similar, but more elaborate argument applies; (see Theorem III.2.).

\section{Acknowledgements}

A.P. thanks G.M. Graf for useful discussions. 


\section{Multiscale virial argument and photon bound}

\section{II.1 Definitions}

We define dilatation operators on the one-particle space $\mathfrak{h}$, constrained to suitable ranges of frequencies, by

$$
d_{n}:=\frac{1}{2} \chi_{n}(|\vec{k}|)\left[\vec{k} \cdot i \vec{\nabla}_{\vec{k}}+i \vec{\nabla}_{\vec{k}} \cdot \vec{k}\right] \chi_{n}(|\vec{k}|)
$$

where $\chi_{n}(|\vec{k}|), n \in \mathbb{N}$, are nonnegative, $C^{\infty}\left(\mathbb{R}^{+}\right)$functions with the properties

(i) $\chi_{n}(|\vec{k}|)=0$, for $|\vec{k}| \leq \frac{1}{2(n+1)}$ and for $|\vec{k}| \geq \frac{3}{2 n}$,

(ii) $\chi_{n}(|\vec{k}|)=1$ for $\frac{1}{n+1} \leq|\vec{k}| \leq \frac{1}{n}$,

(iii) $\left|\chi_{n}^{\prime}(|\vec{k}|)\right| \leq C_{\chi} n$, for all $n \in \mathbb{N}$, where the constant $C_{\chi}$ is independent of $n$.

We also introduce a regularized dilatation operator, $d_{n}^{\epsilon}$, on $\mathfrak{h}$, by setting

$$
d_{n}^{\epsilon}:=\frac{1}{2} \chi_{n}(|\vec{k}|)\left[\vec{k} \cdot i \vec{\nabla}_{\vec{k}}^{\epsilon}+i \vec{\nabla}_{\vec{k}}^{\epsilon} \cdot \vec{k}\right] \chi_{n}(|\vec{k}|)
$$

where $\vec{\nabla} \vec{k}, \epsilon>0$, is the regularized gradient

$$
\vec{\nabla} \epsilon=\frac{\vec{\nabla}_{\vec{k}}}{1-\epsilon^{2} \Delta_{\vec{k}}} .
$$

The operators $d_{n}^{\epsilon}$ are bounded. In the limit $\epsilon \searrow 0$, the action of $d_{n}^{\epsilon}$ on the $j^{\text {th }}$ component of the $\vec{x}$-dependent form factor $\vec{G}_{\vec{x}}^{\alpha}$ is given by

$$
\begin{aligned}
i d_{n} G_{j, \vec{x}}^{\alpha}(\vec{k}, l):= \\
=\quad-\chi_{n}(|\vec{k}|)\left(\vec{k} \cdot \vec{\nabla}_{\vec{k}} \chi_{n}(|\vec{k}|)\right) \frac{\alpha^{3 / 2}}{(2 \pi)^{3 / 2}} \frac{\Lambda(\vec{k})}{\sqrt{2|k|}} P_{j, l}^{\perp}(\vec{k}) e^{-i \alpha \vec{k} \cdot \vec{x}} \\
\quad-\frac{\alpha^{3 / 2}}{(2 \pi)^{3 / 2}} \chi_{n}^{2}(|\vec{k}|)\left(\vec{k} \cdot \overrightarrow{\nabla_{\vec{k}}} \frac{\Lambda(\vec{k})}{\sqrt{2|k|}} P_{j, l}^{\perp}(\vec{k})\right) e^{-i \alpha \vec{k} \cdot \vec{x}} \\
\quad-\frac{1}{2} \chi_{n}^{2}(|\vec{k}|)\left(\vec{\nabla}_{\vec{k}} \cdot \vec{k}\right) \frac{\alpha^{3 / 2}}{(2 \pi)^{3 / 2}} \frac{\Lambda(\vec{k})}{\sqrt{2|k|}} e^{-i \alpha \vec{k} \cdot \vec{x}} P_{j, l}^{\perp}(\vec{k}) \\
\quad-\frac{\alpha^{3 / 2}}{(2 \pi)^{3 / 2}} \chi_{n}^{2}(|\vec{k}|) \frac{\Lambda(\vec{k})}{\sqrt{2|k|}} P_{j, l}^{\perp}(\vec{k})\left(\vec{k} \cdot \vec{\nabla}_{\vec{k}} e^{-i \alpha \vec{k} \cdot \vec{x}}\right) .
\end{aligned}
$$


For later use, the following inequalities should be noted.

$$
\begin{aligned}
|(I I .5)| & \leq \mathcal{O}\left(\alpha^{\frac{3}{2}}\left(n|\vec{k}|^{\frac{1}{2}} \chi_{n}(|\vec{k}|)\right),\right. \\
|(I I .6)|+|(I I .7)|+|(I I .8)| & \leq \mathcal{O}\left(\alpha^{\frac{3}{2}}(|\vec{x}|+1)|\vec{k}|^{-\frac{1}{2}} \chi_{n}(|\vec{k}|)^{2}\right),
\end{aligned}
$$

and

$$
\begin{aligned}
|\operatorname{Im}(I I .5)| & \leq \mathcal{O}\left(\alpha^{\frac{3}{2}} n(|\vec{x}|+1)|\vec{k}|^{\frac{3}{2}} \chi_{n}(|\vec{k}|)\right) \\
|\operatorname{Im}(I I .6)|+|\operatorname{Im}(I I .7)|+|\operatorname{Im}(I I .8)| & \leq \mathcal{O}\left(\alpha^{\frac{3}{2}}(|\vec{x}|+1)|\vec{k}|^{\frac{1}{2}} \chi_{n}(|\vec{k}|)^{2}\right) .
\end{aligned}
$$

Next, we introduce the second quantized operators $D_{n}:=d \Gamma\left(d_{n}\right)$ and $D_{n}^{\epsilon}:=d \Gamma\left(d_{n}^{\epsilon}\right)$. In passing, we also define

$$
\begin{aligned}
H_{n}^{f} & :=\sum_{l=1,2,3} \int d^{3} k a_{l}^{*}(\vec{k}) \chi_{n}(|\vec{k}|)|\vec{k}| \chi_{n}(|\vec{k}|) a_{l}(\vec{k}), \\
N_{n}^{f} & :=\sum_{l=1,2,3} \int d^{3} k a_{l}^{*}(\vec{k}) \chi_{n}(|\vec{k}|)^{2} a_{l}(\vec{k}),
\end{aligned}
$$

for all $n \in \mathbb{N}$.

\section{II.2 Virial theorem}

We fix an $\alpha$-independent, arbitrarily small constant $\Delta_{\Sigma}>0$, and assume that an eigenvector, $\psi_{\alpha}$, corresponding to an eigenvalue in the open interval $\left(E_{g s}, \Sigma-\Delta_{\Sigma}\right)$, exists. We propose to prove a virial theorem allowing us to estimate the expectation value of the photon number operators $N_{n}^{f}$ - scale by scale, i.e., for each $n \in \mathbb{N}$ - in the eigenvector $\psi_{\alpha}$. Our estimates will prove the bound

$$
\left(\psi_{\alpha}, N^{f} \psi_{\alpha}\right)<\mathcal{O}\left(\alpha^{3}\right),
$$

see Theorem II.1.. We remind the reader that, since $\psi_{\alpha} \in P_{H_{\alpha}<\Sigma-\Delta_{\Sigma}} \mathcal{H}$, where $P_{H_{\alpha}<\Sigma-\Delta_{\Sigma}}$ is the projection onto the subspace of vectors with spectral support below $\Sigma-\Delta_{\Sigma}$ w.r.t. the operator $H_{\alpha}$, we have that

$$
\left\||\vec{x}|^{m} \psi_{\alpha}\right\|<\mathcal{O}(1) \quad, \quad \forall m \in \mathbb{N},
$$

where the constant only depends on the choice of $\Delta_{\Sigma}>0$ and on $m$; see $[3],[12]$.

Below, we will give a rigorous justification for the virial identity

$$
\begin{aligned}
0= & \left(\psi_{\alpha}, i\left[H_{\alpha}, D_{n}\right] \psi_{\alpha}\right)= \\
= & \left(\psi_{\alpha}, d \Gamma\left(i\left[|\vec{k}|, d_{n}\right]\right) \psi_{\alpha}\right) \\
& -\left(\psi_{\alpha}, \vec{v} \cdot\left[a^{*}\left(i d_{n} \vec{G}_{\vec{x}}^{\alpha}\right)+a\left(i d_{n} \vec{G}_{\vec{x}}^{\alpha}\right)\right] \psi_{\alpha}\right) \\
& -\left(\psi_{\alpha},\left[a^{*}\left(i d_{n} \vec{G}_{\vec{x}}^{\alpha}\right)+a\left(i d_{n} \vec{G}_{\vec{x}}^{\alpha}\right)\right] \cdot \vec{v} \psi_{\alpha}\right) .
\end{aligned}
$$


For $\epsilon>0$, the following identity holds

$$
\begin{aligned}
0= & \left(\psi_{\alpha},\left[i H_{\alpha}, D_{n}^{\epsilon}\right] \psi_{\alpha}\right) \\
= & \left(\psi_{\alpha}, d \Gamma\left(i\left[|\vec{k}|, d_{n}^{\epsilon}\right]\right) \psi_{\alpha}\right) \\
& -\left(\psi_{\alpha}, \vec{v} \cdot\left[a^{*}\left(i d_{n}^{\epsilon} \vec{G}_{\vec{x}}^{\alpha}\right)+a\left(i d_{n}^{\epsilon} \vec{G}_{\vec{x}}\right)\right] \psi_{\alpha}\right) \\
& -\left(\psi_{\alpha},\left[a^{*}\left(i d_{n}^{\epsilon} \vec{G}_{\vec{x}}^{\alpha}\right)+a\left(i d_{n}^{\epsilon} \vec{G}_{\vec{x}}^{\alpha}\right)\right] \cdot \vec{v} \psi_{\alpha}\right)
\end{aligned}
$$

In (II.18), the right hand side is meaningful because

$$
\psi_{\alpha} \in \mathcal{D}\left(H_{\alpha}\right) \Rightarrow \psi_{\alpha} \in \mathcal{D}\left(H^{f}\right) \Rightarrow \psi_{\alpha} \in \mathcal{D}\left(D_{n}^{\epsilon}\right) .
$$

The terms (II.19), (II.20), and (II.21) are well defined because, thanks to the fact that $\psi_{\alpha} \in P_{H_{\alpha}<\Sigma-\Delta_{\Sigma}} \mathcal{H}$, the following norms are finite:

$$
\begin{aligned}
& \left\|\frac{1}{|\vec{x}|+1} a\left(i d_{n}^{\epsilon} \vec{G}_{\vec{x}}^{\alpha}\right) \psi_{\alpha}\right\|, \\
& \left\||\vec{x}| v_{l} \psi_{\alpha}\right\| .
\end{aligned}
$$

Furthermore, for $\epsilon>0$, (II.18) holds because $\psi_{\alpha}$ is assumed to be an eigenvector of $H_{\alpha}$. Thus, it is enough to prove that the right hand side converges to the right hand side of (II.17), as $\epsilon$ tends to 0 . This can be shown by adapting arguments in [8] (for Nelson's model) to the present model. We note that

ia)

$$
\frac{1}{|\vec{x}|+1}\left\|i d_{n}^{\epsilon} \vec{G}_{\vec{x}}^{\alpha}-i d_{n} \vec{G}_{\vec{x}}^{\alpha}\right\|_{L^{2}\left(\mathbb{R}^{3} ; d^{3} k\right)} \rightarrow 0
$$

as $\epsilon \rightarrow 0$, uniformly in $\vec{x} \in \mathbb{R}^{3}$;

ib) the norm

$$
\left\|\frac{1}{|\vec{x}|+1} a\left(i d_{n}^{\epsilon} \vec{G}_{\vec{x}}^{\alpha}\right) \frac{1}{N_{n}^{f}}\right\|
$$

is uniformly bounded in $\epsilon$;

iia)

$$
i\left[|\vec{k}|, d_{n}^{\epsilon}\right] \rightarrow|\vec{k}| \chi_{n}(|\vec{k}|)^{2}
$$

as $\epsilon \rightarrow 0$, strongly on a dense subset of the one-photon Hilbert space $\mathfrak{h}$; and 
iib) the operator norms

$$
\begin{aligned}
& \left\|\left[|\vec{k}|, d_{n}^{\epsilon}\right]\right\|_{\mathfrak{h}} \\
& \left\|d \Gamma\left(i\left[|\vec{k}|, d_{n}^{\epsilon}\right]\right) \frac{1}{N_{n}^{f}}\right\|
\end{aligned}
$$

are bounded uniformly in $\epsilon$.

Since $\mathcal{D}\left(N_{n}^{f}\right) \cap \mathcal{D}\left(H_{\alpha}\right)=\mathcal{D}\left(H_{\alpha}\right)$ for all $n \in \mathbb{N}$, properties ia) and ib) imply that

$$
\begin{aligned}
& \left(\psi_{\alpha}, \vec{v} \cdot\left[a^{*}\left(i d_{n}^{\epsilon} \vec{G}_{\vec{x}}^{\alpha}\right)+a\left(i d_{n}^{\epsilon} \vec{G}_{\vec{x}}^{\alpha}\right)\right] \psi_{\alpha}\right) \rightarrow \\
& \quad \rightarrow\left(\psi_{\alpha}, \vec{v} \cdot\left[a^{*}\left(i d_{n} \vec{G}_{\vec{x}}^{\alpha}\right)+a\left(i d_{n} \vec{G}_{\vec{x}}^{\alpha}\right)\right] \psi_{\alpha}\right),
\end{aligned}
$$

as $\epsilon \rightarrow 0$, and an analogous result holds for the expression in (II.21);

From iia) and iib), we conclude that

$$
\begin{aligned}
s-\lim _{\epsilon \rightarrow 0} d \Gamma\left(i\left[|\vec{k}|, d_{n}^{\epsilon}\right]\right) \psi_{\alpha} & =d \Gamma\left(i\left[|\vec{k}|, d_{n}\right]\right) \psi_{\alpha} \\
& =H_{n}^{f} \psi_{\alpha}
\end{aligned}
$$

where $H_{n}^{f}$ has been defined in (II.13).

Theorem II.1. Assume that $\psi_{\alpha}$ is a normalized eigenvector of $H_{\alpha}$, with $\psi_{\alpha} \in P_{H<\Sigma-\Delta_{\Sigma}} \mathcal{H}$. Then

$$
\left|\left(\psi_{\alpha}, N^{f} \psi_{\alpha}\right)\right| \leq \mathcal{O}\left(\alpha^{3}\right) .
$$

Proof.

We have shown in (II.17)

$$
0=\left(\psi_{\alpha}, H_{n}^{f} \psi_{\alpha}\right)-\left(\psi_{\alpha},\left[\vec{v} \cdot\left(a^{*}\left(i d_{n} \vec{G}_{\vec{x}}^{\alpha}\right)+a\left(i d_{n} \vec{G}_{\vec{x}}^{\alpha}\right)\right)+\text { h.c. }\right] \psi_{\alpha}\right) .
$$

In order to analyze the right hand side of (II.35), we first calculate some formal commutators.

(i)

$$
\begin{aligned}
& {\left[H_{\alpha}, a\left(i d_{n} G_{j, \vec{x}}^{\alpha}\right)\right]=} \\
& =v_{l}\left[v_{l}, a\left(i d_{n} G_{j, \vec{x}}^{\alpha}\right)\right]+\left[v_{l}, a\left(i d_{n} G_{j, \vec{x}}^{\alpha}\right)\right] v_{l} \\
& \quad+\left[H^{f}, a\left(i d_{n} G_{j, \vec{x}}^{\alpha}\right)\right] .
\end{aligned}
$$

(ii)

$$
\begin{aligned}
& {\left[v_{l}, a\left(i d_{n} G_{j, \vec{x}}^{\alpha}\right)\right]=} \\
& \quad=a\left(i \frac{\partial}{\partial x_{l}}\left(i d_{n} G_{j, \vec{x}}^{\alpha}\right)\right)-\alpha^{\frac{3}{2}} \int \Lambda(\vec{k}) P_{l, l^{\prime}}^{\perp}(\vec{k}) e^{-i \alpha \vec{k} \cdot \vec{x}} \frac{\overline{i\left(d_{n} G_{j, \vec{x}}^{\alpha}\right)\left(\vec{k}, l^{\prime}\right)}}{(2 \pi)^{\frac{3}{2}}|2 \vec{k}|^{\frac{1}{2}}} d^{3} k
\end{aligned}
$$




$$
\begin{aligned}
& {\left[v_{l}, a^{*}\left(i d_{n} G_{j, \vec{x}}^{\alpha}\right)\right]=} \\
& \quad=a^{*}\left(-i \frac{\partial}{\partial x_{l}}\left(i d_{n} G_{j, \vec{x}}^{\alpha}\right)\right)+\alpha^{\frac{3}{2}} \int \Lambda(\vec{k}) P_{l, l^{\prime}}^{\perp}(\vec{k}) e^{i \alpha \vec{k} \cdot \vec{x}} \frac{i\left(d_{n} G_{j, \vec{x}}^{\alpha}\right)\left(\vec{k}, l^{\prime}\right)}{(2 \pi)^{\frac{3}{2}}|2 \vec{k}|^{\frac{1}{2}}} d^{3} k
\end{aligned}
$$

(iii)

$$
\begin{aligned}
& {\left[H^{f}, a\left(i d_{n} G_{j, \vec{x}}^{\alpha}\right)\right]=-a\left(|\vec{k}| i d_{n} G_{j, \vec{x}}^{\alpha}\right)} \\
& {\left[H^{f}, a^{*}\left(i d_{n} G_{j, \vec{x}}^{\alpha}\right)\right]=a^{*}\left(|\vec{k}| i d_{n} G_{j, \vec{x}}^{\alpha}\right) .}
\end{aligned}
$$

Then we use the identity

$$
\vec{v}=-\frac{i}{2}\left[\vec{x}, H_{\alpha}\right]
$$

which holds in the sense of quadratic forms on vectors belonging to $\mathcal{D}\left(H_{\alpha}\right) \cap$ $\mathcal{D}(|\vec{x}|)$. Making use of the commutators (i),(ii), (iii), and thanks to our assumption that $\psi_{\alpha} \in P_{H_{\alpha}<\Sigma-\Delta_{\Sigma}} \mathcal{H}$, we can write

$$
\begin{aligned}
\left(\psi_{\alpha}\right. & \left., \vec{v} \cdot a\left(i d_{n} \vec{G}_{\vec{x}}^{\alpha}\right) \psi_{\alpha}\right)= \\
& =\frac{i}{2}\left(\psi_{\alpha},\left[H_{\alpha}, \vec{x}\right] \cdot a\left(i d_{n} \vec{G}_{\vec{x}}^{\alpha}\right) \psi_{\alpha}\right) \\
& =-\frac{i}{2}\left(\psi_{\alpha}, \vec{x} \cdot\left[H_{\alpha}, a\left(i d_{n} \vec{G}_{\vec{x}}^{\alpha}\right)\right] \psi_{\alpha}\right),
\end{aligned}
$$

hence

$$
\begin{aligned}
\left(\psi_{\alpha},\right. & \left.\vec{v} \cdot a\left(i d_{n} \vec{G}_{\vec{x}}^{\alpha}\right) \psi_{\alpha}\right)= \\
= & \frac{i}{2}\left(\psi_{\alpha}, x_{j} a\left(|\vec{k}| i d_{n} G_{j, \vec{x}}^{\alpha}\right) \psi_{\alpha}\right) \\
& +\frac{i \alpha^{\frac{3}{2}}}{2}\left(\psi_{\alpha}, x_{j} v_{l} \int \Lambda(\vec{k}) P_{l, l^{\prime}}^{\perp}(\vec{k}) e^{-i \alpha \vec{k} \cdot \vec{x}} \frac{\frac{i\left(d_{n} G_{j, \vec{x}}^{\alpha}\right)\left(\vec{k}, l^{\prime}\right)}{(2 \pi)^{\frac{3}{2}}|2 \vec{k}|^{\frac{1}{2}}}}{\left.d^{3} k \psi_{\alpha}\right)}\right. \\
& +\frac{i \alpha^{\frac{3}{2}}}{2}\left(\psi_{\alpha}, x_{j} \int \Lambda(\vec{k}) P_{l, l^{\prime}}^{\perp}(\vec{k}) e^{-i \alpha \vec{k} \cdot \vec{x}} \frac{\frac{i\left(d_{n} G_{j, \vec{x}}^{\alpha}\right)\left(\vec{k}, l^{\prime}\right)}{(2 \pi)^{\frac{3}{2}}|2 \vec{k}|^{\frac{1}{2}}}}{d^{3} k} v_{l} \psi_{\alpha}\right) \\
& -i\left(\psi_{\alpha}, x_{j} v_{l} a\left(i \frac{\partial}{\partial x_{l}}\left(i d_{n} G_{j, \vec{x}}^{\alpha}\right)\right) \psi_{\alpha}\right) \\
& -\frac{i}{2}\left(\psi_{\alpha}, x_{j}\left[a\left(i \frac{\partial}{\partial x_{l}}\left(i d_{n} G_{j, \vec{x}}^{\alpha}\right)\right), v_{l}\right] \psi_{\alpha}\right) .
\end{aligned}
$$


Similarly,

$$
\begin{aligned}
\left(\psi_{\alpha}, \vec{v} \cdot a^{*}\left(i d_{n} \vec{G}_{\vec{x}}^{\alpha}\right) \psi_{\alpha}\right)= & \\
= & -\frac{i}{2}\left(\psi_{\alpha}, x_{j} a^{*}\left(|\vec{k}| i d_{n} G_{j, \vec{x}}^{\alpha}\right) \psi_{\alpha}\right) \\
& -\frac{i \alpha^{\frac{3}{2}}}{2}\left(\psi_{\alpha}, x_{j} v_{l} \int \Lambda(\vec{k}) P_{l, l^{\prime}}^{\perp}(\vec{k}) e^{i \alpha \vec{k} \cdot \vec{x}} \frac{i d_{n} G_{j, \vec{x}}^{\alpha}\left(\vec{k}, l^{\prime}\right)}{(2 \pi)^{\frac{3}{2}}|2 \vec{k}|^{\frac{1}{2}}} d^{3} k \psi_{\alpha}\right) \\
& -\frac{i \alpha^{\frac{3}{2}}}{2}\left(\psi_{\alpha}, x_{j} \int \Lambda(\vec{k}) P_{l, l^{\prime}}^{\perp}(\vec{k}) e^{i \alpha \vec{k} \cdot \vec{x}} \frac{i d_{n} G_{j, \vec{x}}^{\alpha}\left(\vec{k}, l^{\prime}\right)}{(2 \pi)^{\frac{3}{2}}|2 \vec{k}|^{\frac{1}{2}}} d^{3} k v_{l} \psi_{\alpha}\right) \\
& -i\left(\psi_{\alpha}, a^{*}\left(-i \frac{\partial}{\partial x_{l}}\left(i d_{n} G_{j, \vec{x}}^{\alpha}\right) x_{j} v_{l} \psi_{\alpha}\right) .\right. \\
& -\frac{i}{2}\left(\psi_{\alpha}, x_{j}\left[v_{l}, a^{*}\left(-i \frac{\partial}{\partial x_{l}}\left(i d_{n} G_{j, \vec{x}}^{\alpha}\right)\right] \psi_{\alpha}\right) .\right.
\end{aligned}
$$

To bound the terms appearing in the expressions above, with the expected $n$-dependence, it is convenient to suitably separate them into two groups.

We separately treat the terms - said to be of type I - containing the creationand annihilation operators smeared out with derivatives w.r. to $\vec{x}$ of the function $d_{n} \vec{G}_{\vec{x}}^{\alpha}(\vec{k})$, or with $d_{n} \vec{G}_{\vec{x}}^{\alpha}(\vec{k})$ multiplied by $|\vec{k}|$, and the terms - said to be of type $I I$ - where they only appear in the velocity operator.

Thus, we first analyze the expressions (II.50),(II.53),(II.56) and (II.59). Similar arguments will apply to terms arising from the commutators in (II.54), and (II.60).

Afterwards, we will analyze the terms (II.51),(II.52),(II.57) and (II.58), and those arising from the commutators in (II.54),(II.60), which do not contain field variables.

Terms of type I

In estimating norms of the type

$$
\left\|a\left(|\vec{k}| i d_{n} G_{j, \vec{x}}^{\alpha}\right) \psi_{\alpha}\right\|
$$

we make use of the inequalities in (II.9), (II.10):

$$
\begin{aligned}
& \mid\left(\psi_{\alpha},\right.\left.x_{j} a\left(|\vec{k}| i d_{n} G_{j, \vec{x}}^{\alpha}\right) \psi_{\alpha}\right) \mid \leq \\
& \leq \alpha^{\frac{3}{2}} c_{1}\left\||\vec{x}| \psi_{\alpha}\right\|\left(n^{2} \int \mathbf{1}_{\left(\frac{1}{2(n+1)}, \frac{3}{2 n}\right)}(\vec{k})|\vec{k}|^{3} d^{3} k\right)^{\frac{1}{2}}\left(\psi_{\alpha}, N_{n}^{f} \psi_{\alpha}\right)^{\frac{1}{2}} \\
& \quad+\alpha^{\frac{3}{2}} c_{2}\left\||\vec{x}|^{2} \psi_{\alpha}\right\|\left(\int \mathbf{1}_{\left(\frac{1}{2(n+1)}, \frac{3}{2 n}\right)}(\vec{k})|\vec{k}| d^{3} k\right)^{\frac{1}{2}}\left(\psi_{\alpha}, N_{n}^{f} \psi_{\alpha}\right)^{\frac{1}{2}}
\end{aligned}
$$

where $c_{1}$ and $c_{2}$ are $n$ - and $\alpha$-independent, and $\mathbf{1}_{\left(\frac{1}{2(n+1)}, \frac{3}{2 n}\right)}(\vec{k})$ is the characteristic function of the set

$$
\left\{\vec{k} \in \mathbb{R}^{3}, \frac{1}{2(n+1)}<|\vec{k}|<\frac{3}{2 n}\right\} .
$$


Similar estimates can be derived for the terms in Eqs. (II.53), (II.59), and for those of the same type that result from the commutators in Eqs. (II.54), (II.60).

Terms of type II

To analyze expressions (II.51),(II.52),(II.57) and (II.58), we first observe that the contributions proportional to

$$
\operatorname{Re}\left(e^{-i \alpha \vec{k} \cdot \vec{x}} \overline{i\left(d_{n} G_{j, \vec{x}}^{\alpha}\right)\left(\vec{k}, l^{\prime}\right)}\right)=\operatorname{Re}\left(e^{i \alpha \vec{k} \cdot \vec{x}} i\left(d_{n} G_{j, \vec{x}}^{\alpha}\right)\left(\vec{k}, l^{\prime}\right)\right)
$$

cancel. The contributions corresponding to the imaginary parts can be estimated individually as follows

$$
\begin{aligned}
\mid \frac{i \alpha^{\frac{3}{2}}}{2}\left(\psi_{\alpha}, x_{j} v_{l} \int\right. & \left.i \operatorname{Im}\left(P_{l, l^{\prime}}^{\perp}(\vec{k}) e^{-i \alpha \vec{k} \cdot \vec{x}} \frac{\overline{i\left(d_{n} G_{j, \vec{x}}^{\alpha}\right)\left(\vec{k}, l^{\prime}\right)}}{|\vec{k}|^{\frac{1}{2}}}\right) d^{3} k \psi_{\alpha}\right) \mid \leq \\
\leq & \alpha^{3} c_{1}^{\prime \prime} \sum_{l}\left\||\vec{x}| v_{l}|\vec{x}| \psi_{\alpha}\right\|\left(\int \mathbf{1}_{\left(\frac{1}{2}(n+1), \frac{3}{2} n\right)}(\vec{k}) d^{3} k\right) \\
& +\alpha^{3} c_{2}^{\prime \prime} \sum_{l}\left\||\vec{x}| v_{l}|\vec{x}| \psi_{\alpha}\right\|\left(n \int \mathbf{1}_{\left(\frac{1}{2}(n+1), \frac{3}{2} n\right)}(\vec{k})|\vec{k}| d^{3} k\right),
\end{aligned}
$$

where $c_{1}^{\prime \prime}$ and $c_{2}^{\prime \prime}$ are $n$ - and $\alpha$-independent constants; here we have exploited inequalities (II.11), (II.12).

Similar estimates can be proven for other terms arising from the commutators in Eqs. (II.54), (II.60).

To prove Theorem II.1., the following ingredients must be used:

(1) $\left\||\vec{x}| v_{l}|\vec{x}| \psi_{\alpha}\right\|,\left\|v_{l}|\vec{x}| \psi_{\alpha}\right\|$ and $\left\||\vec{x}| \psi_{\alpha}\right\|$ are uniformly bounded in $\alpha$, because $\psi_{\alpha} \in P_{H_{\alpha}<\Sigma-\Delta_{\Sigma}} \mathcal{H}$

(2) the crucial scaling behavior of the bounds

$$
\begin{aligned}
& n^{2}\left(n^{2} \int \mathbf{1}_{\left(\frac{1}{2(n+1)}, \frac{3}{2 n}\right)}(\vec{k})|\vec{k}|^{3} d^{3} k\right)^{\frac{1}{2}} \leq \mathcal{O}(1) \\
& n^{2}\left(\int \mathbf{1}_{\left(\frac{1}{2(n+1)}, \frac{3}{2 n}\right)}(\vec{k})|\vec{k}| d^{3} k\right)^{\frac{1}{2}} \leq \mathcal{O}(1) \\
& n^{3}\left(\int \mathbf{1}_{\left(\frac{1}{2(n+1)}, \frac{3}{2 n}\right)}(\vec{k}) d^{3} k\right) \leq \mathcal{O}(1) \\
& n^{3}\left(n \int \mathbf{1}_{\left(\frac{1}{2(n+1)}, \frac{3}{2 n}\right)}(\vec{k})|\vec{k}| d^{3} k\right) \leq \mathcal{O}(1) .
\end{aligned}
$$

Returning to (II.35) and using the bounds just proven, we can write

$$
0 \geq\left(\psi_{\alpha}, H_{n}^{f} \psi_{\alpha}\right)-\alpha^{\frac{3}{2}} Q_{1} \frac{1}{n^{2}}\left(\psi_{\alpha}, N_{n}^{f} \psi_{\alpha}\right)^{\frac{1}{2}}-\alpha^{3} Q_{2} \frac{1}{n^{3}},
$$


for some $n-$ and $\alpha$-independent positive constants $Q_{1}$ and $Q_{2}$. Due to the support of $\chi_{n}(|\vec{k}|)$, we have

$$
\left(\psi_{\alpha}, H_{n}^{f} \psi_{\alpha}\right) \geq \frac{1}{2(n+1)}\left(\psi_{\alpha}, N_{n}^{f} \psi_{\alpha}\right)
$$

hence, from the inequality (II.74), we derive the bound

$$
n^{2}\left(\psi_{\alpha}, N_{n}^{f} \psi_{\alpha}\right) \leq \mathcal{O}\left(\alpha^{3}\right)
$$

for all $n \in \mathbb{N}$. These estimates enable us to bound the expectation value of the photon number operator in $\psi_{\alpha}$ :

$$
N^{f}=N_{(1,+\infty)}^{f}+\sum_{n=1}^{\infty} N_{\left(\frac{1}{n+1}, \frac{1}{n}\right)}^{f},
$$

where $N_{(a, b)}^{f}:=\sum_{l=1,2,3} \int d^{3} k a_{l}^{*}(\vec{k}) \mathbf{1}_{(a, b)}(|\vec{k}|) a_{l}(\vec{k}) ;$ namely

$$
\begin{aligned}
& \left(\psi_{\alpha}, N^{f} \psi_{\alpha}\right)= \\
& \quad=\left(\psi_{\alpha}, N_{(1,+\infty)}^{f} \psi_{\alpha}\right)+\sum_{n=1}^{\infty}\left(\psi_{\alpha}, N_{\left(\frac{1}{n+1}, \frac{1}{n}\right)}^{f} \psi_{\alpha}\right) \\
& \quad \leq\left(\psi_{\alpha}, N_{0}^{f} \psi_{\alpha}\right)+\sum_{n=1}^{\infty}\left(\psi_{\alpha}, N_{n}^{f} \psi_{\alpha}\right) \\
& \quad \leq \mathcal{O}\left(\alpha^{3}\right)
\end{aligned}
$$

where

$$
N_{0}^{f}:=\sum_{l=1,2,3} \int d^{3} k a_{l}^{*}(\vec{k}) \chi_{0}(|\vec{k}|)^{2} a_{l}(\vec{k})
$$

with $\chi_{0}(|\vec{k}|)$ a nonnegative, $C^{\infty}\left(\mathbb{R}^{+}\right)$function such that $\chi_{0}(\vec{k})=0$ for $|\vec{k}| \leq \frac{1}{2}$ and $\chi_{0}(\vec{k})=1$ for $|\vec{k}| \geq 1$. By the same virial argument, the estimate $\left(\psi_{\alpha}, N_{0}^{f} \psi_{\alpha}\right) \leq \mathcal{O}\left(\alpha^{3}\right)$ follows.

This completes our proof of Theorem II.1..

\section{Absence of excited eigenstates of $H_{\alpha}$}

We start this section with an important technical lemma on properties of eigenvalues and eigenstates of the Hamiltonian $H_{\alpha}$. In Theorem II.1., we have seen that, if $\psi_{\alpha}$ is a normalized eigenvector of $H_{\alpha}$ corresponding to an eigenvalue $E_{\alpha}, E_{\alpha} \leq \Sigma-\Delta_{\Sigma}, \Delta_{\Sigma}>0$, then

$$
\left(\psi_{\alpha}, N^{f} \psi_{\alpha}\right) \leq C_{N^{f}} \alpha^{3}
$$


where $C_{N^{f}}$ only depends on the distance between the eigenvalue $E_{\alpha}$ and the ionization threshold, $\Sigma$, of the unperturbed system.

Lemma III.1. Let $\Delta_{\Sigma}>0$, and assume that $|\alpha|<\bar{\alpha}\left(\Delta_{\Sigma}\right)$, for a sufficiently small $\bar{\alpha}\left(\Delta_{\Sigma}\right)>0$. Let $E_{\alpha}$ be an eigenvalue of $H_{\alpha}$ in the interval $\left(E_{g s}(\alpha), \Sigma-\Delta_{\Sigma}\right)$ corresponding to an eigenvector $\psi_{\alpha}$. Then the eigenvector $\psi_{\alpha}$, with $\left\|\psi_{\alpha}\right\|=1$, and $E_{\alpha}$ can be written as

$$
\begin{aligned}
& \psi_{\alpha}=P_{\Omega} \otimes P_{\mathcal{H}_{e l}^{(j)}} \psi_{\alpha}+P_{\Omega} \otimes P_{\mathcal{H}_{e l}^{(j)}}^{\perp} \psi_{\alpha}+P_{\Omega}^{\perp} \otimes \mathbf{1}_{\mathcal{H}_{e l}} \psi_{\alpha}, \\
& E_{\alpha}=E^{(j)}+\Delta E_{\alpha}^{(j)}
\end{aligned}
$$

where $P_{\mathcal{H}^{\prime}}$ is the projection onto a subspace $\mathcal{H}^{\prime}$ of a Hilbert space $\tilde{\mathcal{H}}$, $P_{\mathcal{H}^{\prime}}^{\perp}$ is the orthogonal projection onto the orthogonal complement of $\mathcal{H}^{\prime}$ in $\tilde{\mathcal{H}}$, and $P_{\Omega}=P_{\{\mathbb{C} \Omega\}}$. Furthermore, for some $j \geq 0$, the terms on the right hand side of (III.2) and (III.3) obey the following bounds:

$$
\begin{aligned}
\left\|P_{\Omega} \otimes P_{\mathcal{H}_{e l}^{(j)}} \psi_{\alpha}\right\|^{2} & \geq 1-B_{j} \alpha^{3} \\
\left\|P_{\Omega}^{\perp} \otimes \mathbf{1}_{\mathcal{H}_{e l}} \psi_{\alpha}\right\| & \leq C_{N^{f}}^{\frac{1}{2}} \alpha^{\frac{3}{2}} \\
\left\|P_{\Omega} \otimes P_{\mathcal{H}_{e l}^{(j)}}^{\perp} \psi_{\alpha}\right\| & \leq C_{j}^{\perp} \alpha^{3} \\
\left|\Delta E_{\alpha}^{(j)}\right| & \leq C_{E^{(j)}} \alpha^{3}
\end{aligned}
$$

and the constants $B_{j}, C_{j}^{\perp}$, and $C_{E^{(j)}}$ depend only on the distance of $E^{(j)}$ from the rest of the spectrum of the atomic Hamiltonian $H_{e l}$.

Proof.

We divide the interval $\left(E_{g s}(\alpha), \Sigma-\Delta_{\Sigma}\right)$ into $\bar{i}+1$ intervals, where $\bar{i}$ is the number of excited energy levels of the atomic Hamiltonian $H_{e l}$ contained in the open interval $\left(E_{g s}(\alpha), \Sigma-\Delta_{\Sigma}\right)$, for some arbitrary, but fixed, $\Delta_{\Sigma}>0$. We define the subintervals

$$
\begin{aligned}
I_{0} & :=\left(E_{g s}(\alpha), E^{(0)}+\frac{\sigma_{1,0}}{2}\right), \\
I_{i} & :=\left[E^{(i)}-\frac{\sigma_{i, i-1}}{2}, E^{(i)}+\frac{\sigma_{i+1, i}}{2}\right) \quad 1 \leq i<\bar{i}-1, \\
I_{\bar{i}} & :=\left[E^{(\bar{i})}-\frac{\sigma_{\bar{i}, \bar{i}-1}}{2}, \Sigma-\Delta_{\Sigma}\right),
\end{aligned}
$$

where $\sigma_{i, i-1}:=E^{(i)}-E^{(i-1)}$. Henceforth, the feinstructure constant $\alpha$ is assumed to be so small that $E^{(0)}+\frac{\sigma_{1,0}}{2}>E_{g s}(\alpha)$.

Since $E_{\alpha}<\Sigma-\Delta_{\Sigma}, E_{\alpha}$ belongs to one of the intervals $I_{i}, 0 \leq i \leq \bar{i} ; I_{j}$, say. We will show that it is, in fact, as close to $E^{(j)}$ as we wish if $\alpha$ is chosen sufficiently small. 
Any vector $\psi_{\alpha}$ can be written as in Eq. (III.2). Thus, we only have to prove the bounds in (III.4),(III.5),(III.6) and (III.7).

Inequality (III.5) is a straightforward consequence of Theorem II.1. In fact, since $E_{\alpha}<\Sigma-\Delta_{\Sigma}$, by assumption, the electron in the state $\psi_{\alpha}$ is exponentially well localized near the nucleus. Thus, the assumptions in Theorem II.1 are satisfied, and we have the bound

$$
\left\|P_{\Omega}^{\perp} \otimes \mathbf{1}_{\mathcal{H}_{e l}} \psi_{\alpha}\right\|^{2} \leq\left(\psi_{\alpha}, N^{f} \psi_{\alpha}\right) \leq C_{N^{f}} \alpha^{3},
$$

for some $\alpha$-independent constant $C_{N^{f}}$ only depending on $\Sigma-\Delta_{\Sigma}$.

To prove the remaining bounds, we consider the eigenvalue equation, $H_{\alpha} \psi_{\alpha}=E_{\alpha} \psi_{\alpha}$, from which we derive the following identities.

i) Let $H_{I}:=H_{\alpha}-H_{0}$, and let $\mathcal{P}_{j}^{ \pm}$denote the projection $P_{\Omega} \otimes P_{\mathcal{H}_{e l}^{(j)}}^{\perp, \pm}$, where $P_{\mathcal{H}_{e l}^{(j)}}^{\perp, \pm}$ are the spectral projections onto the subspaces of $\mathcal{H}_{e l}$ orthogonal to $\mathcal{H}_{e l}^{(j)}$ and with spectral support w.r. to $H_{e l}$ above and below $E^{(j)}$, respectively. Then

$$
\begin{aligned}
H_{0} \mathcal{P}_{j}^{ \pm} \psi_{\alpha}= & E_{\alpha} \mathcal{P}_{j}^{ \pm} \psi_{\alpha}-\mathcal{P}_{j}^{ \pm} H_{I} P_{\Omega}^{\perp} \otimes \mathbf{1}_{\mathcal{H}_{e l}} \psi_{\alpha} \\
& -\left\langle H_{I}\right\rangle_{\Omega} \mathcal{P}_{j}^{ \pm} \psi_{\alpha},
\end{aligned}
$$

where $\left\langle H_{I}\right\rangle_{\Omega}$ is a multiple of the identity obtained by Wick-ordering $H_{I}$. Note that if $j=0$ only $\mathcal{P}_{0}^{+}$is present.

ii) Moreover,

$$
\begin{aligned}
P_{\Omega}^{\perp} \otimes & \mathbf{1}_{\mathcal{H}_{e l}} H_{\alpha} P_{\Omega}^{\perp} \otimes \mathbf{1}_{\mathcal{H}_{e l}} \psi_{\alpha}= \\
= & -P_{\Omega}^{\perp} \otimes \mathbf{1}_{\mathcal{H}_{e l}} H_{I}\left(P_{\Omega} \otimes P_{\mathcal{H}_{e l}^{(j)}} \psi_{\alpha}+P_{\Omega} \otimes P_{\mathcal{H}_{e l}^{(j)}}^{\perp} \psi_{\alpha}\right) \\
\quad & +E_{\alpha} P_{\Omega}^{\perp} \otimes \mathbf{1}_{\mathcal{H}_{e l}} \psi_{\alpha} .
\end{aligned}
$$

From Eq. (III.12), we derive the equation

$$
\mathcal{P}_{j}^{ \pm} \psi_{\alpha}=-\frac{1}{H_{0}-E_{\alpha}+\left\langle H_{I}\right\rangle_{\Omega}} \mathcal{P}_{j}^{ \pm} H_{I} P_{\Omega}^{\perp} \otimes \mathbf{1}_{\mathcal{H}_{e l}} \psi_{\alpha}
$$

Note that the R.S. of (III.14) is well defined for $\alpha$ so small that

$$
\left|\left\langle H_{I}\right\rangle_{\Omega}\right|=\frac{\alpha^{3}}{(2 \pi)^{3}} \int \frac{d^{3} k}{2|\vec{k}|} \Lambda(\vec{k}) P_{i, l}^{\perp}(\vec{k}) P_{i, l}^{\perp}(\vec{k}) d^{3} k<\min \left\{\frac{\sigma_{j+1, j}}{2}, \frac{\sigma_{j, j-1}}{2}\right\} .
$$


Using (III.1), it is then straightforward to derive the bound

$$
\left\|\mathcal{P}_{j}^{ \pm} \psi_{\alpha}\right\| \leq C_{j}^{\perp} \alpha^{3},
$$

where the constant $C_{j}^{\perp}$ depends on $C_{N^{f}}$ and on the energy differences $\sigma_{j, j-1}, \sigma_{j+1, j}$. This proves (III.6). From (III.2), (III.1), and (III.16) we then conclude that

$$
\left(\psi_{\alpha}, \psi_{\alpha}\right)=1 \leq\left\|P_{\Omega} \otimes P_{\mathcal{H}_{e l}^{(j)}} \psi_{\alpha}\right\|^{2}+\left(C_{j}^{\perp}\right)^{2} \alpha^{6}+C_{N^{f}} \alpha^{3},
$$

which implies that

$$
1 \geq\left|c_{j}(\alpha)\right|^{2}:=\left\|P_{\Omega} \otimes P_{\mathcal{H}_{e l}^{(j)}} \psi_{\alpha}\right\|^{2} \geq 1-B_{j} \alpha^{3}
$$

for some $B_{j}$ independent of $\alpha$. This proves (III.4).

Next, we estimate the difference $E_{\alpha}-E^{(j)}$ starting from the eigenvalue equation $E_{\alpha}=\left\langle\psi_{\alpha}, H_{\alpha} \psi_{\alpha}\right\rangle$, with $\psi_{\alpha}$ given by (III.2). Thus

$$
\begin{aligned}
E_{\alpha}= & \left(E^{(j)}+\left\langle H_{I}\right\rangle_{\Omega}\right)\left|c_{j}(\alpha)\right|^{2} \\
& +\left[\left(P_{\Omega} \otimes P_{\mathcal{H}_{e l}^{(j)}} \psi_{\alpha}, H_{I} P_{\Omega}^{\perp} \otimes \mathbf{1}_{\mathcal{H}_{e l}} \psi_{\alpha}\right)+c . c .\right] \\
& +\left(P_{\Omega} \otimes P_{\mathcal{H}_{e l}^{(j)}}^{\perp} \psi_{\alpha}, H_{\alpha} P_{\Omega} \otimes P_{\mathcal{H}_{e l}^{(j)}}^{\perp} \psi_{\alpha}\right) \\
& +\left[\left(P_{\Omega}^{\perp} \otimes \mathbf{1}_{\mathcal{H}_{e l}} \psi_{\alpha}, H_{I} P_{\Omega} \otimes P_{\mathcal{H}_{e l}^{(j)}}^{\perp} \psi_{\alpha}\right)+c . c .\right] \\
& +\left(P_{\Omega}^{\perp} \otimes \mathbf{1}_{\mathcal{H}_{e l}} \psi_{\alpha}, H_{\alpha} P_{\Omega}^{\perp} \otimes \mathbf{1}_{\mathcal{H}_{e l}} \psi_{\alpha}\right),
\end{aligned}
$$

where we have used that $P_{\Omega} \otimes \mathbf{1}_{\mathcal{H}_{e l}} H_{0} P_{\Omega}^{\perp} \otimes \mathbf{1}_{\mathcal{H}_{e l}}=0$. The different terms, (III.20) - (III.23), on the R.S. are bounded as follows.

1) Bound on (III.20):

$$
\begin{aligned}
& \left|\left(P_{\Omega} \otimes P_{\mathcal{H}_{e l}^{(j)}} \psi_{\alpha}, H_{I} P_{\Omega}^{\perp} \otimes \mathbf{1}_{\mathcal{H}_{e l}} \psi_{\alpha}\right)\right| \leq \\
& \leq 2 \alpha^{\frac{3}{2}}\left|\left(P_{\Omega} \otimes P_{\mathcal{H}_{e l}^{(j)}} \psi_{\alpha}, \vec{p} \cdot \vec{A}(\alpha \vec{x}) P_{\Omega}^{\perp} \otimes \mathbf{1}_{\mathcal{H}_{e l}} \psi_{\alpha}\right)\right| \\
& +\alpha^{3}\left|\left(P_{\Omega} \otimes P_{\mathcal{H}_{e l}^{(j)}} \psi_{\alpha}, \vec{A}(\alpha \vec{x}) \cdot \vec{A}(\alpha \vec{x}) P_{\Omega}^{\perp} \otimes \mathbf{1}_{\mathcal{H}_{e l}} \psi_{\alpha}\right)\right|( \\
& \leq D_{1} \alpha^{\frac{3}{2}}\left|c_{j}(\alpha)\right|\left\|P_{\Omega}^{\perp} \otimes \mathbf{1}_{\mathcal{H}_{e l}} \psi_{\alpha}\right\| \leq \tilde{D}_{1} \alpha^{3}
\end{aligned}
$$

where $D_{1}$ only depends on $j$.

2) Bound on (III.21): To estimate, e.g., the contribution to (III.21) pro- 
portional to $H_{0}$, we use Eq. (III.14) as follows.

$$
\begin{gathered}
\left.\sum_{ \pm} \mid\left(P_{\Omega} \otimes P_{\mathcal{H}_{e l}^{(j)}}^{\perp, \pm} \psi_{\alpha}, H_{0} P_{\Omega} \otimes P_{\mathcal{H}_{e l}^{(j)}}^{\perp, \pm} \psi_{\alpha}\right)\right) \mid= \\
=\sum_{ \pm} \mid\left(\frac{1}{H_{0}-E_{\alpha}+\left\langle H_{I}\right\rangle_{\Omega}} \mathcal{P}_{j}^{ \pm} H_{I} P_{\Omega}^{\perp} \otimes \mathbf{1}_{\mathcal{H}_{e l}^{(j)}} \psi_{\alpha},\right. \\
\left.\quad, H_{0} \frac{1}{H_{0}-E_{\alpha}+\left\langle H_{I}\right\rangle_{\Omega}} \mathcal{P}_{j}^{ \pm} H_{I} P_{\Omega}^{\perp} \otimes \mathbf{1}_{\mathcal{H}_{e l}^{(j)}} \psi_{\alpha}\right) \mid \\
\leq D_{2}\left\|\left(N^{f}\right)^{\frac{1}{2}} P_{\Omega}^{\perp} \otimes \mathbf{1}_{\mathcal{H}_{e l}^{(j)}} \psi_{\alpha}\right\|^{2} \leq \tilde{D}_{2} \alpha^{3},
\end{gathered}
$$

where $D_{2}$ depends on the maximum of the following operator norms

$$
\left\|R_{1} \mathcal{P}_{j}^{ \pm}\left(\frac{1}{H_{0}-E_{\alpha}-\left\langle H_{I}\right\rangle_{\Omega}}\right)^{2} H_{0} \mathcal{P}_{j}^{ \pm} R_{2}\right\|,
$$

where the operators $R_{1}, R_{2}$ can be either a component of $\vec{p}$ or a component of $\vec{A}(\alpha \vec{x})$. These norms are bounded uniformly in $\alpha$.

The expectation value of $H_{I}$ in (III.21) is bounded similarly.

3) Bound on (III.22): Using Eq. (III.14) we find that

$$
\begin{gathered}
\mid\left(P_{\Omega}^{\perp} \otimes \mathbf{1}_{\left.\mathcal{H}_{e l} \psi_{\alpha}, H_{I} P_{\Omega} \otimes P_{\mathcal{H}_{e l}^{(j)}}^{\perp} \psi_{\alpha}\right) \mid \leq}\right. \\
\leq \sum_{ \pm}\left|\left(P_{\Omega}^{\perp} \otimes \mathbf{1}_{\mathcal{H}_{e l}} \psi_{\alpha}, H_{I} \frac{1}{H_{0}-E_{\alpha}+\left\langle H_{I}\right\rangle_{\Omega}} \mathcal{P}_{j}^{ \pm} H_{I} P_{\Omega}^{\perp} \otimes \mathbf{1}_{\mathcal{H}_{e l}} \psi_{\alpha}\right)\right| \leq \tilde{D}_{3} \alpha^{3}
\end{gathered}
$$

4) Bound on (III.23): We use the eigenvalue equation and (III.13) to find that

$$
\begin{aligned}
\mid\left(P_{\Omega}^{\perp} \otimes\right. & \left.\mathbf{1}_{\mathcal{H}_{e l}} \psi_{\alpha}, H_{\alpha} P_{\Omega}^{\perp} \otimes \mathbf{1}_{\mathcal{H}_{e l}} \psi_{\alpha}\right) \mid= \\
= & \left|\left(P_{\Omega}^{\perp} \otimes \mathbf{1}_{\mathcal{H}_{e l}} \psi_{\alpha}, P_{\Omega}^{\perp} \otimes \mathbf{1}_{\mathcal{H}_{e l}} H_{\alpha} P_{\Omega}^{\perp} \otimes \mathbf{1}_{\mathcal{H}_{e l}} \psi_{\alpha}\right)\right| \\
\leq & \left|\left(P_{\Omega}^{\perp} \otimes \mathbf{1}_{\mathcal{H}_{e l}} \psi_{\alpha}, H_{I}\left(P_{\Omega} \otimes P_{\mathcal{H}_{e l}^{j}} \psi_{\alpha}+P_{\Omega} \otimes P_{\mathcal{H}_{e l}^{(j)}}^{\perp} \psi_{\alpha}\right)\right)\right| \\
& +\left|E_{\alpha}\right|\left|\left(P_{\Omega}^{\perp} \otimes \mathbf{1}_{\mathcal{H}_{e l}} \psi_{\alpha}, P_{\Omega}^{\perp} \otimes \mathbf{1}_{\mathcal{H}_{e l}} \psi_{\alpha}\right)\right| \\
\leq & \left|\left(P_{\Omega}^{\perp} \otimes \mathbf{1}_{\mathcal{H}_{e l}} \psi_{\alpha}, H_{I}\left(P_{\Omega} \otimes P_{\mathcal{H}_{e l}^{(j)}} \psi_{\alpha}\right)\right)\right| \\
& +\left|\sum_{ \pm}\left(P_{\Omega}^{\perp} \otimes \mathbf{1}_{\mathcal{H}_{e l}} \psi_{\alpha}, H_{I} \frac{1}{H_{0}-E_{\alpha}+\left\langle H_{I}\right\rangle_{\Omega}} \mathcal{P}_{j}^{ \pm} H_{I} P_{\Omega}^{\perp} \otimes \mathbf{1}_{\mathcal{H}_{e l}^{(j)}} \psi_{\alpha}\right)\right|(I) I \\
& +\left|E_{\alpha}\right|\left|\left(P_{\Omega}^{\perp} \otimes \mathbf{1}_{\mathcal{H}_{e l}} \psi_{\alpha}, P_{\Omega}^{\perp} \otimes \mathbf{1}_{\mathcal{H}_{e l}} \psi_{\alpha}\right)\right| \leq \tilde{D}_{4} \alpha^{3}
\end{aligned}
$$

To conclude the proof of (III.7) we use (III.19)-(III.23) and exploit the bounds (III.11),(III.16) and (III.18) to show that there is a constant $C_{E^{(j)}}$ depending 
on $\Sigma-E^{(j)}$ and on the energy shifts $\sigma_{j, j-1}, \sigma_{j+1, j}$, but uniform in $\alpha$, such that

$$
\left|E_{\alpha}-E^{(j)}\right|=\left|\Delta E_{\alpha}^{(j)}\right| \leq C_{E^{(j)}} \alpha^{3}
$$

We are now in a position to show that an eigenvector $\psi_{\alpha}$ corresponding to an eigenvalue $E_{\alpha} \in I_{j}$ cannot exist for $\alpha$ small enough, where our estimate of the upper bound on $|\alpha|$ depends on the interval $I_{j}$.

Theorem III.2. Let $\psi_{\alpha}$ be an arbitrary normalized vector. Assume that there exists some $j$, with $j \leq \bar{i}$, such that the bounds in Eqs. (III.4),(III.5),(III.6), and (III.7) hold. Then, for $\alpha$ small enough, $\psi_{\alpha}$ cannot be solution of the eigenvalue equation $H_{\alpha} \psi_{\alpha}=E_{\alpha} \psi_{\alpha}$ if $\kappa>\left|E^{(0)}\right|$, where $\kappa$ is the u.v. cutoff.

Proof.

Our proof is indirect. Thus, we assume that $\psi_{\alpha}$ obeys the eigenvalue equation with $\left\|\psi_{\alpha}\right\|=1$. We also assume that $\kappa>\left|E^{(0)}\right|$. We distinguish three cases, $j>1, j=1$ and $j=0$.

Case $j>1$

Because of Property (P1) stated in the Introduction, see Eq. (I.22), there exists a dipole transition from the eigenstate $\phi^{(j)}$ of $H_{e l}$ to a lower-energy eigenstate $\phi^{(i)}$. More precisely, there is an eigenvector, $\Omega \otimes \phi^{(i)},\left\|\phi^{(i)}\right\|=1$, $\phi^{(i)} \in \mathcal{H}_{e l}^{(i)}$, of $H_{e l}$ such that

$$
\theta_{l}(\hat{k})\left(\phi^{(i)}, p_{l^{\prime}} P_{l, l^{\prime}}^{\perp}(\vec{k}) \phi^{(j)}\right)=\theta_{l}(\hat{k})\left(\phi^{(i)}, p_{l} \phi^{(j)}\right) \neq 0,
$$

for some functions $\theta_{l}(\hat{k}), k_{l} \theta_{l}(\hat{k})=0$, peaked at some $\overline{\hat{k}}:=\frac{\vec{k}}{|\vec{k}|}$, where $\phi^{(j)}$ is such that

$$
\Omega \otimes \phi^{(j)}=\frac{P_{\Omega} \otimes P_{\mathcal{H}_{e l}^{(j)}} \psi_{\alpha}}{\left\|P_{\Omega} \otimes P_{\mathcal{H}_{e l}^{(j)}} \psi_{\alpha}\right\|} .
$$

We then consider the vector $\eta^{(i)} \in \mathcal{F}_{\text {phys. }}$

$$
\eta^{(i)}:=\int d^{3} k \frac{1}{\epsilon^{\frac{1}{2}}} h\left(\frac{|\vec{k}|-E_{\alpha}+E^{(i)}}{\epsilon}\right) \theta_{l}(\hat{k}) a_{l}^{*}(\vec{k}) \Omega \otimes \phi^{(i)}
$$

where $\epsilon>0$, and $h(z) \in C_{0}^{\infty}(\mathbb{R}), h(0)=1$.

The eigenvalue equation for $\psi_{\alpha}$ implies that

$$
\left(\eta^{(i)},\left(H_{0}-E_{\alpha}\right) \psi_{\alpha}\right)=-\left(\eta^{(i)}, H_{I} \psi_{\alpha}\right)
$$


By Lemma III.1., this equation implies that

$\left(\eta^{(i)},\left(H_{0}-E_{\alpha}\right) P_{\Omega}^{\perp} \otimes \mathbf{1}_{\mathcal{H}_{e l}} \psi_{\alpha}\right)+2 \alpha^{\frac{3}{2}}\left(\eta^{(i)}, \vec{p} \cdot \vec{A}(\alpha \vec{x}) P_{\Omega} \otimes P_{\mathcal{H}_{e l}^{(j)}} \psi_{\alpha}\right)=\mathcal{O}\left(\alpha^{3}\right)$,

with $P_{\Omega} \otimes P_{\mathcal{H}_{e l}^{(j)}} \psi_{\alpha}=: c_{j}(\alpha) \Omega \otimes \phi^{(j)}$. We now notice that

$$
\begin{aligned}
& \left(\eta^{(i)}, \vec{p} \cdot \vec{A}(\alpha \vec{x}) \Omega \otimes \phi^{(j)}\right)= \\
& \quad=\int d^{3} k \frac{1}{\epsilon^{\frac{1}{2}}} h\left(\frac{|\vec{k}|-E_{\alpha}+E^{(i)}}{\epsilon}\right) \theta_{l}(\hat{k}) \frac{\Lambda(\vec{k})}{|2 \vec{k}|^{\frac{1}{2}}}\left(\phi^{(i)}, p_{l^{\prime}} P_{l, l^{\prime}}^{\perp}(\vec{k}) e^{-i \alpha \vec{k} \cdot \vec{x}} \phi^{(j)}\right) .
\end{aligned}
$$

Hence, using (III.42) we see that, for $\epsilon$ and $\alpha$ small enough, the following bound holds

$$
2\left|\left(\eta^{(i)}, \vec{p} \cdot \vec{A}(\alpha \vec{x}) \Omega \otimes \phi^{(j)}\right)\right|>Q_{1} \epsilon^{\frac{1}{2}},
$$

where $Q_{1}$ is an $\epsilon$ - and $\alpha$ - independent constant.

By (III.1), we have that

$$
\begin{aligned}
& \left|\left(\eta^{(i)},\left(H_{0}-E_{\alpha}\right) P_{\Omega}^{\perp} \otimes \mathbf{1}_{\mathcal{H}_{e l}} \psi_{\alpha}\right)\right| \leq \\
& \quad \leq\left\|\left(H_{0}-E_{\alpha}\right) \eta^{(i)}\right\|\left\|P_{\Omega}^{\perp} \otimes \mathbf{1}_{\mathcal{H}_{e l}} \psi_{\alpha}\right\| \\
& \quad \leq C_{N^{f}}^{\frac{1}{2}} \alpha^{\frac{3}{2}}\left\|\left(H_{0}-E_{\alpha}\right) \eta^{(i)}\right\|,
\end{aligned}
$$

and

$$
\begin{aligned}
& \left\|\left(H_{0}-E_{\alpha}\right) \eta^{(i)}\right\|= \\
= & {\left[\sum_{l=1,2,3} \int d^{3} k \frac{1}{\epsilon}\left|h\left(\frac{|\vec{k}|-E_{\alpha}+E^{(i)}}{\epsilon}\right) \theta_{l}(\hat{k})\right|^{2}\left(|\vec{k}|-E_{\alpha}+E^{(i)}\right)^{2}\right]^{\frac{1}{2}} } \\
\leq & Q_{2} \epsilon,
\end{aligned}
$$

where $Q_{2}$ is an $\epsilon$ - and $\alpha$ - independent constant. Inequalities (III.47), (III.50) and (III.51) imply that

$$
Q_{1} \epsilon^{\frac{1}{2}} \leq Q_{2} \epsilon+\mathcal{O}\left(\alpha^{\frac{3}{2}}\right)
$$

which yields a contradiction if we choose $\epsilon$ proportional to $\alpha$, and $\alpha$ is small enough.

$\frac{\text { Case } j=1}{\text { If } j=1}$ in Eq. (III.42), has angular momentum $l \neq 0$. But if $\phi^{(j=1)}$ has angular momentum $l=0$, i.e., $\phi^{(j=1)} \equiv \phi_{l=0}^{(j=1)}$, the matrix element in (III.42) vanishes, because of the selection rule that forbids transitions with $l^{\prime}-l \neq \pm 1$, where 
$l^{\prime}$ and $l$ are the orbital angular momenta of the final and of the initial state, respectively.

Therefore, in this particular case, we are forced to consider two-photon transitions.

We then construct a suitable vector $\xi^{(0)} \in \mathcal{H}$ yielding a contradiction to the assumption that $\psi_{\alpha}$ obeys the eigenvalue equation.

Choice of $\xi^{(0)}$.

$$
\begin{gathered}
\xi^{(0)}:=\iint d^{3} k d^{3} q \frac{1}{\epsilon^{\frac{1}{2}}} h_{1}\left(\frac{|\vec{k}|+|\vec{q}|+\alpha^{3} f(|\vec{k}|+|\vec{q}|)-E_{\alpha}+E^{(0)}}{\epsilon}\right) h_{2}(|\vec{k}|-|\vec{q}|) \times \\
\times \theta_{j}(\hat{k}) \theta_{j^{\prime}}(\hat{q}) a_{j}^{*}(\vec{k}) a_{j^{\prime}}^{*}(\vec{q}) \Omega \otimes \phi^{(0)},
\end{gathered}
$$

where:

- $h_{1}(z) \in C_{0}^{\infty}((-1,1))$ and $h_{1}(0)=1$;

- $h_{2}(z) \geq 0$ has support in

$$
\left(E_{\alpha}-E^{(0)}-\epsilon-4 \delta, E_{\alpha}-E^{(0)}+\epsilon-2 \delta\right),
$$

with $0<\epsilon, \alpha \ll \delta \ll 1$ and $\alpha^{4} \leq \epsilon$; thus,

$$
|\vec{k}|+|\vec{q}| \geq E_{\alpha}-E^{(0)}-\epsilon-4 \delta ;
$$

- for $z=|\vec{k}|+|\vec{q}|$, we define

$$
\begin{aligned}
& f(z):= \\
= & -\frac{4}{(2 \pi)^{3}} \int\left(\phi^{(0)}, p_{i} P_{i, j}^{\perp}(\vec{r}) e^{-i \alpha \vec{r} \cdot \vec{x}} \frac{1}{H_{e l}+z+|\vec{r}|-E_{\alpha}} e^{i \alpha \vec{r} \cdot \vec{x}} P_{j, i^{\prime}}^{\perp}(\vec{r}) p_{i^{\prime}} \phi^{(0)}\right) \frac{\Lambda(\vec{r})^{2} d^{3} r}{2|\vec{r}|} \\
& +\frac{\left\langle H_{I}\right\rangle}{\alpha^{3}} .
\end{aligned}
$$

Note that, being $\alpha, \epsilon$ and $\delta$ small enough, and thanks to the constraint in (III.54),

$$
|f(z)| \leq K
$$

and

$$
c_{1} \geq 1+\alpha^{3} \frac{d f(z)}{d z} \geq c_{2}>0,
$$

for constants $K, c_{1}$, and $c_{2}$ uniform in $\alpha$ and $\epsilon$; this follows because

$$
\left|E_{\alpha}-E^{(1)}\right| \leq \mathcal{O}\left(\alpha^{3}\right)
$$

and

$$
P_{\mathcal{H}_{e l}^{(0)}} e^{i \alpha \vec{r} \cdot \vec{x}} P_{j, i^{\prime}}^{\perp}(\vec{r}) p_{i^{\prime}} \phi^{(0)}=0
$$


- the angular functions $\theta_{j}(\hat{k}), j=1,2,3, k_{j} \theta_{j}(\hat{k})=0$, are peaked at some $\overline{\hat{k}}$.

We derive some constraints on the values of $|\vec{k}|$ and $|\vec{q}|$, that follow from our definitions of the functions $h_{1}, h_{2}$ : The two inequalities

$$
E_{\alpha}-E^{(0)}-\epsilon \leq|\vec{k}|+|\vec{q}|+\alpha^{3} f(|\vec{k}|+|\vec{q}|) \leq E_{\alpha}-E^{(0)}+\epsilon
$$

and

$$
E_{\alpha}-E^{(0)}-\epsilon-4 \delta \leq|\vec{k}|-|\vec{q}| \leq E_{\alpha}-E^{(0)}+\epsilon-2 \delta
$$

imply that

$$
E_{\alpha}-E^{(0)}-\epsilon-2 \delta \leq|\vec{k}|+\mathcal{O}\left(\alpha^{3}\right) \leq E_{\alpha}-E^{(0)}+\epsilon-\delta
$$

and

$$
\delta-2 \epsilon \leq|\vec{q}|+\mathcal{O}\left(\alpha^{3}\right) \leq 2 \delta+2 \epsilon .
$$

Similarly to the dipole case, we are going to consider the nonvanishing matrix elements below

$$
\begin{aligned}
& \theta_{j}(\hat{w}) \theta_{j^{\prime}}\left(\hat{w^{\prime}}\right)\left(\phi^{(0)}, p_{l^{\prime}} P_{l^{\prime}, j}^{\perp}(\vec{w}) \frac{1}{H_{e l}-E_{\alpha}+\left|\overrightarrow{w^{\prime}}\right|} p_{l^{\prime \prime}} P_{l^{\prime \prime}, j^{\prime}}^{\perp}\left(\overrightarrow{w^{\prime}}\right) \phi_{l=0}^{(1)}\right)=(\mathrm{I} \\
= & \theta_{j}(\hat{w}) \theta_{j^{\prime}}\left(\hat{w^{\prime}}\right)\left(\phi^{(0)}, p_{j} \frac{1}{H_{e l}-E_{\alpha}+\left|\overrightarrow{w^{\prime}}\right|} p_{j^{\prime}} \phi_{l=0}^{(1)}\right) \\
= & \sum_{j} \theta_{j}(\hat{w}) \theta_{j}\left(\hat{w}^{\prime}\right)\left(\phi^{(0)}, p_{j} \frac{1}{H_{e l}-E_{\alpha}+\left|\overrightarrow{w^{\prime}}\right|} p_{j} \phi_{l=0}^{(1)}\right)
\end{aligned}
$$

with $\vec{w}, \overrightarrow{w^{\prime}}$ either equal to $\vec{k}, \vec{q}$ or to $\vec{q}, \vec{k}$, respectively, with the constraints specified in (III.60) and (III.61). The step from (III.63) to (III.64) follows because the wave functions representing the vectors $\phi^{(0)}$ and $\phi_{l=0}^{(1)}$ are rotationally invariant.

The eigenvalue equation for $\psi_{\alpha}$ implies that

$$
\left(\xi^{(0)},\left(H_{0}-E_{\alpha}\right) \psi_{\alpha}\right)=-\left(\xi^{(0)}, H_{I} \psi_{\alpha}\right) .
$$

The term on the right hand side is given by

$$
-2 \alpha^{\frac{3}{2}}\left(\xi^{(0)}, \vec{p} \cdot \vec{A}(\alpha \vec{x}) \psi_{\alpha}\right)-\alpha^{3}\left(\xi^{(0)},(\vec{A}(0)+\Delta \vec{A}(\alpha \vec{x}))^{2} \psi_{\alpha}\right),
$$

where $\Delta \vec{A}(\alpha \vec{x}):=\vec{A}(\alpha \vec{x})-\vec{A}(0)$. Thus

$$
\begin{aligned}
& \left(\xi^{(0)},\left(H_{0}-E_{\alpha}\right) \psi_{\alpha}\right)= \\
= & -2 \alpha^{\frac{3}{2}}\left(\xi^{(0)}, \vec{p} \cdot \vec{A}(\alpha \vec{x}) \psi_{\alpha}\right)-\alpha^{3}\left(\xi^{(0)}, \vec{A}(0)^{2} \psi_{\alpha}\right) \\
& -2 \alpha^{3}\left(\xi^{(0)}, \vec{A}(0) \cdot \Delta \vec{A}(\alpha \vec{x}) \psi_{\alpha}\right) \\
& -\alpha^{3}\left(\xi^{(0)}, \Delta \vec{A}(\alpha \vec{x})^{2} \psi_{\alpha}\right) .
\end{aligned}
$$


For the moment, we just observe that the absolute values of the terms in (III.69), (III.70) are bounded above by $\mathcal{O}\left(\alpha^{5}\right)$. For the term (III.70), the claim is evident. To bound the term (III.69), it is enough to notice that the first order term in the expansion in $\alpha$ of $\Delta \vec{A}(\alpha \vec{x})$ does not give any contribution not bounded by $\mathcal{O}\left(\alpha^{5}\right)$, thanks to the selection rule for the angular momentum;(hint: the first order term in the expansion of

$$
\left(\xi^{(0)}, \vec{A}(0) \cdot \Delta \vec{A}(\alpha \vec{x}) \phi_{l=0}^{(1)} \otimes \Omega\right)
$$

vanishes). Thus, we conclude that

$$
\begin{aligned}
\left(\xi^{(0)},\right. & \left.\left(H_{0}-E_{\alpha}\right) \psi_{\alpha}\right)= \\
= & -2 \alpha^{\frac{3}{2}}\left(\xi^{(0)}, \vec{p} \cdot \vec{A}(\alpha \vec{x}) \psi_{\alpha}\right) \\
& -\alpha^{3}\left(\xi^{(0)}, \vec{A}(0)^{2} \psi_{\alpha}\right) \\
& +\mathcal{O}\left(\alpha^{5}\right) .
\end{aligned}
$$

We then need to analyze terms (III.72) and (III.73). To bound (III.72), we start by noticing that

$$
\begin{aligned}
& -2 \alpha^{\frac{3}{2}}\left(\xi^{(0)}, \vec{p} \cdot \vec{A}(\alpha \vec{x}) \psi_{\alpha}\right) \\
= & -2 \alpha^{\frac{3}{2}}\left(\xi^{(0)}, \vec{p} \cdot \vec{A}(\alpha \vec{x}) \frac{1}{H_{0}-E_{\alpha}}\left(H_{0}-E_{\alpha}\right) \psi_{\alpha}\right) \\
= & 2 \alpha^{\frac{3}{2}}\left(\xi^{(0)}, \vec{p} \cdot \vec{A}(\alpha \vec{x}) \frac{1}{H_{0}-E_{\alpha}} H_{I} \psi_{\alpha}\right) .
\end{aligned}
$$

The step from line (III.75) to line (III.76) is legitimate because:

(i)

$$
\mathbf{1}_{\mathcal{F}_{\text {phys. }}} \otimes P_{\mathcal{H}_{\text {el }}^{(0)}} \vec{p} \cdot \vec{A}(\alpha \vec{x}) \xi^{(0)}=0 ;
$$

this follows from the fact that the angular momentum of $\phi^{(0)}$ is 0 and $\vec{A}$ is transverse.

(ii) By inequalities (III.60), (III.61),

$$
|\vec{k}| \geq|\vec{q}| \geq \mathcal{O}(\delta)>0 .
$$

Consequently, the denominator of $\frac{1}{H_{0}-E_{\alpha}}$, in the given expression, takes values larger than a positive quantity of order $\mathcal{O}(\delta)$. (We recall that $\left|E^{(1)}-E_{\alpha}\right| \leq$ $\left.\mathcal{O}\left(\alpha^{3}\right)\right)$ 
Next, we replace $H_{I}$, in (III.77), by $2 \alpha^{\frac{3}{2}} \vec{p} \cdot \vec{A}(\alpha \vec{x})$, discarding a term that can be bounded above by $\mathcal{O}\left(\alpha^{6}\right)$. To see this, we note that, for the term proportional to $\vec{A}(\alpha \vec{x})^{2}$, the lowest-order contribution is given by

$$
\begin{aligned}
& 2 \alpha^{\frac{3}{2}} \alpha^{3}\left(\xi^{(0)}, \vec{p} \cdot \vec{A}(\alpha \vec{x}) \frac{1}{H_{0}-E_{\alpha}} \vec{A}(0) \vec{A}(0) \psi_{\alpha}\right)= \\
= & 2 \alpha^{\frac{3}{2}} \alpha^{3}\left(\xi^{(0)}, \vec{p} \cdot \vec{A}(\alpha \vec{x}) \frac{1}{H_{0}-E_{\alpha}} \vec{A}(0) \vec{A}(0)\left(\psi_{\alpha}-c_{1}(\alpha) \Omega \otimes \phi_{l=0}^{(1)}\right)\right),
\end{aligned}
$$

where $\left.\| \psi_{\alpha}-c_{1}(\alpha) \Omega \otimes \phi^{(1)}\right) \| \leq \mathcal{O}\left(\alpha^{\frac{3}{2}}\right)$; see (III.5) and (III.6). In fact,

$$
\alpha^{\frac{3}{2}} \alpha^{3}\left(\xi^{(0)}, \vec{p} \cdot \vec{A}(\alpha \vec{x}) \frac{1}{H_{0}-E_{\alpha}} \Delta \vec{A}(\alpha \vec{x}) \vec{A}(0) c_{1}(\alpha) \Omega \otimes \phi_{l=0}^{(1)}\right)=0,
$$

because the vector $c_{1}(\alpha) \Omega \otimes \phi_{l=0}^{(1)}$ does not contain any photons.

Thus, we have shown that

$$
(I I I .72)=4 \alpha^{3}\left(\xi^{(0)}, \vec{p} \cdot \vec{A}(\alpha \vec{x}) \frac{1}{H_{0}-E_{\alpha}} \vec{p} \cdot \vec{A}(\alpha \vec{x}) \psi_{\alpha}\right)+\mathcal{O}\left(\alpha^{5}\right) .
$$

We continue by estimating the first term on the right hand side of (III.83). We first consider the contribution

$$
4 \alpha^{3}\left(\xi^{(0)}, \vec{p} \cdot \vec{A}(\alpha \vec{x}) \frac{1}{H_{0}-E_{\alpha}} \vec{p} \cdot \vec{A}(\alpha \vec{x}) c_{1}(\alpha) \Omega \otimes \phi_{l=0}^{(1)}\right),
$$

which can be estimated from below as follows.

In the following we assume the properties of $h_{1}, h_{2}, \theta_{j}, \epsilon, \delta$, and $\alpha$ described above. By using the steps from (III.63) to (III.64), the result proven in Lemma A1 implies that, for some $\mathcal{Q}_{1}>0$ independent of $\alpha$ and $\epsilon$,

$$
\begin{aligned}
& \left|c_{1}(\alpha)\right| \mid \iint \frac{\Lambda(\vec{k})}{|\vec{k}|^{\frac{1}{2}}} \frac{\Lambda(\vec{q})}{|\vec{q}|^{\frac{1}{2}}} \frac{1}{\epsilon^{\frac{1}{2}}} h_{1}\left(\frac{g(|\vec{k}|+|\vec{q}|)-E_{\alpha}+E^{(0)}}{\epsilon}\right) h_{2}(|\vec{k}|-|\vec{q}|) \theta_{j}(\hat{k}) \theta_{j^{\prime}}(\hat{q}) \times \\
& \times\left(\phi^{(0)}, p_{l^{\prime}} P_{l^{\prime}, j}^{\perp}(\vec{q}) \frac{1}{H_{e l}-E_{\alpha}+|\vec{k}|} p_{l^{\prime \prime}} P_{l^{\prime \prime}, j^{\prime}}^{\perp}(\vec{k}) \phi_{l=0}^{(1)}\right) d^{3} q d^{3} k \\
& +\iint \frac{\Lambda(\vec{k})}{|\vec{k}|^{\frac{1}{2}}} \frac{\Lambda(\vec{q})}{|\vec{q}|^{\frac{1}{2}}} \frac{1}{\epsilon^{\frac{1}{2}}} h_{1}\left(\frac{g(|\vec{k}|+|\vec{q}|)-E_{\alpha}+E^{(0)}}{\epsilon}\right) h_{2}(|\vec{k}|-|\vec{q}|) \theta_{j}(\hat{k}) \theta_{j^{\prime}}(\hat{q}) \times \\
& \times\left(\phi^{(0)}, p_{l^{\prime}} P_{l^{\prime}, j}^{\perp}(\vec{k}) \frac{1}{H_{e l}-E_{\alpha}+|\vec{q}|} p_{l^{\prime \prime}} P_{l^{\prime \prime}, j^{\prime}}^{\perp}(\vec{q}) \phi_{l=0}^{(1)}\right) d^{3} q d^{3} k \mid \\
& \geq \mathcal{Q}_{1} \epsilon^{\frac{1}{2}}
\end{aligned}
$$

where

$$
g(z):=z+\alpha^{3} f(z) \quad(z>0) .
$$


Therefore, for some $Q_{1}^{\prime}>0$ independent of $\alpha$ and $\epsilon$, and for $\alpha$ and $\epsilon$ small enough, we have

$$
|(I I I .84)| \geq Q_{1}^{\prime} \epsilon^{\frac{1}{2}} \alpha^{3} .
$$

Notice, moreover, that the contribution corresponding to $\mathcal{P}_{1}^{ \pm} \equiv P_{\Omega} \otimes P_{\mathcal{H}_{e l}^{(1)}}^{\perp \pm}$ in the first term of the R. S. of (III.83) is $\mathcal{O}\left(\alpha^{6}\right)$, thanks to (III.6). Thus we have that

$$
\begin{aligned}
& (I I I .72)= \\
= & (I I I .84) \\
& +4 \alpha^{3}\left(\xi^{(0)}, \vec{p} \cdot \vec{A}(\alpha \vec{x}) \frac{1}{H_{0}-E_{\alpha}} \vec{p} \cdot \vec{A}(\alpha \vec{x}) P_{\Omega}^{\perp} \otimes \mathbf{1}_{\mathcal{H}_{e l}} \psi_{\alpha}\right) \\
+ & \mathcal{O}\left(\alpha^{5}\right),
\end{aligned}
$$

if $\epsilon$ and $\alpha$ are chosen small enough. Our next step is to treat the term in (III.92). For this purpose we note that the possible combinations of photon operators are the following ones:

$$
\begin{aligned}
& 4 \alpha^{3}\left(\xi^{(0)}, \vec{p} \cdot \vec{A}^{(-)}(\alpha \vec{x}) \frac{1}{H_{0}-E_{\alpha}} \vec{p} \cdot \vec{A}^{(+)}(\alpha \vec{x}) P_{\Omega}^{\perp} \otimes \mathbf{1}_{\mathcal{H}_{e l}} \psi_{\alpha}\right), \\
& 4 \alpha^{3}\left(\xi^{(0)}, \vec{p} \cdot \vec{A}^{(+)}(\alpha \vec{x}) \frac{1}{H_{0}-E_{\alpha}} \vec{p} \cdot \vec{A}^{(-)}(\alpha \vec{x}) P_{\Omega}^{\perp} \otimes \mathbf{1}_{\mathcal{H}_{e l}} \psi_{\alpha}\right),
\end{aligned}
$$

and

$$
4 \alpha^{3}\left(\xi^{(0)}, \vec{p} \cdot \vec{A}^{(-)}(\alpha \vec{x}) \frac{1}{H_{0}-E_{\alpha}} \vec{p} \cdot \vec{A}^{(-)}(\alpha \vec{x}) P_{\Omega}^{\perp} \otimes \mathbf{1}_{\mathcal{H}_{e l}} \psi_{\alpha}\right) .
$$

Here, $\vec{A}^{(+)}(\alpha \vec{x}), \vec{A}^{(-)}(\alpha \vec{x})$ denote the contributions to $\vec{A}(\alpha \vec{x})$ proportional to the creation- and the annihilation operators, respectively.

In expression (III.94), we contract the photon operator in $\vec{A}^{(+)}(\alpha \vec{x})$ first with the two photon operators in $\xi^{(0)}$ (term (III.98)) and after with the photon operator in $\vec{A}^{(-)}(\alpha \vec{x})$ (term (III.99)), and we obtain

$$
\begin{aligned}
& =4 \alpha^{3} \overbrace{\xi^{(0)}, \vec{p} \cdot \vec{A}^{(-)}(\alpha \vec{x}) \frac{1}{H_{0}-E_{\alpha}} \vec{p} \cdot \vec{A}^{(+)}}^{\left.(\alpha \vec{x}) P_{\Omega}^{\perp} \otimes \mathbf{1}_{\mathcal{H}_{e l}} \psi_{\alpha}\right)} \\
& \left.\quad+4 \alpha^{3}(\xi^{(0)}, \vec{p} \cdot \vec{A}^{(-)} \overbrace{(\alpha \vec{x}) \frac{1}{H_{0}-E_{\alpha}} \vec{p} \cdot \vec{A}^{(+)}}(\alpha \vec{x})\rangle P_{\Omega}^{\perp} \otimes \mathbf{1}_{\mathcal{H}_{e l}} \psi_{\alpha}\right),
\end{aligned}
$$

where

$$
\vec{p} \cdot \vec{A}^{(-)} \overbrace{(\alpha \vec{x}) \frac{1}{H_{0}-E_{\alpha}} \vec{p} \cdot \vec{A}^{(+)}}(\alpha \vec{x})
$$


stands for the expression

$$
\vec{p} \cdot \vec{A}^{(-)}(\alpha \vec{x}) \frac{1}{H_{0}-E_{\alpha}} \vec{p} \cdot \vec{A}^{(+)}(\alpha \vec{x})
$$

after contracting the operators $\vec{A}^{(-)}(\alpha \vec{x}), \vec{A}^{(+)}(\alpha \vec{x})$.

By standard calculations, the absolute value of (III.95) and (III.98) can be bounded from above by $\mathcal{O}\left(\epsilon^{\frac{1}{2}} \alpha^{3} \alpha^{\frac{3}{2}}\right)$. The factor $\epsilon^{\frac{1}{2}}$ comes from the contraction of $\vec{A}^{(+)}(\alpha \vec{x})$ with one of the two photon creation operators appearing in the expression for the vector $\xi^{(0)}$, the factor $\alpha^{\frac{3}{2}}$ comes from (III.1).

To control the term (III.96), we proceed as follows: In one of the operators $\vec{A}^{(-)}(\alpha \vec{x})$, we split the photon momentum space integral into two contributions corresponding to photon frequencies larger and smaller than $\epsilon^{\frac{1}{4}}$, respectively:

$$
\begin{aligned}
A_{j}^{(-)}(\alpha \vec{x})= & \frac{1}{(2 \pi)^{3 / 2}} \sum_{j^{\prime}=1,2,3} \int_{B_{\epsilon^{1 / 4}}} \frac{d^{3} k}{\sqrt{2|\vec{k}|}} \Lambda(\vec{k}) P_{j, j^{\prime}}^{\perp}(\vec{k}) e^{i \vec{k} \cdot \alpha \vec{x}} a_{j^{\prime}}(\vec{k}) \\
& +\frac{1}{(2 \pi)^{3 / 2}} \sum_{j^{\prime}=1,2,3} \int_{\mathbb{R}^{3} \backslash B_{\epsilon^{1 / 4}}} \frac{d^{3} k}{\sqrt{2|\vec{k}|}} \Lambda(\vec{k}) P_{j, j^{\prime}}^{\perp}(\vec{k}) e^{i \vec{k} \cdot \alpha \vec{x}} a_{j^{\prime}}(\vec{k})
\end{aligned}
$$

where $B_{\epsilon^{1 / 4}}:=\left\{\vec{k} \in \mathbb{R}^{3}:|\vec{k}|<\epsilon^{\frac{1}{4}}\right\}$. We denote the corresponding operators by $\vec{A}_{<}^{(-)}(\alpha \vec{x})$ and $\vec{A}_{>}^{(-)}(\alpha \vec{x})$, respectively.

We first consider the term proportional to $\vec{A}_{<}^{(-)}(\alpha \vec{x})$. Because of the constraint on the frequencies, its contribution to expression (III.96) can be bounded from above by $\mathcal{O}\left(\epsilon^{\frac{1}{8}} \alpha^{3} \alpha^{\frac{3}{2}}\right)$.

To bound the term proportional to $\vec{A}_{>}^{(-)}(\alpha \vec{x})$, we observe that, in the scalar product

$$
4 \alpha^{3}\left(\xi^{(0)}, \vec{p} \cdot \vec{A}_{>}^{(-)}(\alpha \vec{x}) \frac{1}{H_{0}-E_{\alpha}} \vec{p} \cdot \vec{A}^{(-)}(\alpha \vec{x}) P_{\Omega}^{\perp} \otimes \mathbf{1}_{\mathcal{H}_{e l}} \psi_{\alpha}\right),
$$

we can insert $\frac{1}{H_{0}-E_{\alpha}}\left(H_{0}-E_{\alpha}\right)$ again, as follows

$4 \alpha^{3}\left(\xi^{(0)}, \vec{p} \cdot \vec{A}_{>}^{(-)}(\alpha \vec{x}) \frac{1}{H_{0}-E_{\alpha}} \vec{p} \cdot \vec{A}^{(-)}(\alpha \vec{x}) \frac{1}{H_{0}-E_{\alpha}}\left(H_{0}-E_{\alpha}\right) P_{\Omega}^{\perp} \otimes \mathbf{1}_{\mathcal{H}_{e l}} \psi_{\alpha}\right)$.

For $\epsilon$ small enough, this is well defined, because, with respect to the operator $H_{0}-E_{\alpha}$, the vector

$$
\vec{p} \cdot \vec{A}^{(+)}(\alpha \vec{x}) \frac{1}{H_{0}-E_{\alpha}} \vec{p} \cdot \vec{A}_{>}^{(+)}(\alpha \vec{x}) \xi^{(0)}
$$


has spectral support above a positive constant of order $\epsilon^{-\frac{1}{4}}$. To see this, notice that $H_{0}$, applied to $\xi^{(0)}$, takes values larger than

$$
E^{(0)}+\left(E_{\alpha}-E^{(0)}-\epsilon-\mathcal{O}\left(\alpha^{3}\right)\right)
$$

see (III.58). Furthermore the operator $\vec{A}_{>}^{(+)}(\alpha \vec{x})$ yields an additive term of order $\mathcal{O}\left(\epsilon^{\frac{1}{4}}\right)$. It follows that the spectral support of

$$
\vec{p} \cdot \vec{A}^{(+)}(\alpha \vec{x}) \frac{1}{H_{0}-E_{\alpha}} \vec{p} \cdot \vec{A}_{>}^{(+)}(\alpha \vec{x}) \xi^{(0)}
$$

w.r. to the operator $H_{0}-E_{\alpha}$ lies above

$$
\left\{\left[E^{(0)}+\left(E_{\alpha}-E^{(0)}-\epsilon-\mathcal{O}\left(\alpha^{3}\right)\right)+\mathcal{O}\left(\epsilon^{\frac{1}{4}}\right)\right]-E_{\alpha}\right\} \geq \mathcal{O}\left(\epsilon^{\frac{1}{4}}\right) .
$$

In expression (III.104), we may also replace

$$
\left(H_{0}-E_{\alpha}\right) P_{\Omega}^{\perp} \otimes \mathbf{1}_{\mathcal{H}_{e l}} \psi_{\alpha} \quad \text { by } \quad-H_{I} P_{\Omega}^{\perp} \otimes \mathbf{1}_{\mathcal{H}_{e l}} \psi_{\alpha}
$$

because the remainder is given by the vector

$$
-\left(H_{\alpha}-E_{\alpha}\right)\left(c_{1}(\alpha) \Omega \otimes \phi_{l=0}^{(1)}+P_{\Omega} \otimes P_{\mathcal{H}_{e l}^{(1)}}^{\perp} \psi_{\alpha}\right),
$$

which contains only one or two photons, and, hence, does not contribute to expression (III.104). Thus (III.104) can be replaced by

$$
-4 \alpha^{3}\left(\xi^{(0)}, \vec{p} \cdot \vec{A}_{>}^{(-)}(\alpha \vec{x}) \frac{1}{H_{0}-E_{\alpha}} \vec{p} \cdot \vec{A}^{(-)}(\alpha \vec{x}) \frac{1}{H_{0}-E_{\alpha}} H_{I} P_{\Omega}^{\perp} \otimes \mathbf{1}_{\mathcal{H}_{e l}} \psi_{\alpha}\right),
$$

and the absolute value of this quantity can be bounded from above by $\mathcal{O}\left(\epsilon^{-\frac{1}{4}} \alpha^{6}\right)$. This is seen by noticing that there are two powers of $\alpha^{\frac{3}{2}}$ coming from $H_{I}$ and from $P_{\Omega}^{\perp} \otimes \mathbf{1}_{\mathcal{H}_{e l}} \psi_{\alpha}$, respectively, while the denominator (in the second resolvent) is bounded from below by a quantity of order $\epsilon^{\frac{1}{4}}$.

In expression (III.99), we first split (III.99)

$$
\begin{aligned}
& 4 \alpha^{3}(\xi^{(0)}, \vec{p} \cdot \vec{A}^{(-)} \overbrace{(\alpha \vec{x}) \frac{1}{H_{0}-E_{\alpha}} \vec{p} \cdot \vec{A}^{(+)}}(\alpha \vec{x}) \mathbf{1}_{\mathcal{F}_{p h y s}} \otimes P_{\mathcal{H}_{e l}^{(0)}} P_{\Omega}^{\perp} \otimes \mathbf{1}_{\mathcal{H}_{e l}} \psi_{\alpha}) \\
& +4 \alpha^{3}(\xi^{(0)}, \vec{p} \cdot \vec{A}^{(-)} \overbrace{(\alpha \vec{x}) \frac{1}{H_{0}-E_{\alpha}} \vec{p} \cdot \vec{A}^{(+)}}(\alpha \vec{x}) \mathbf{1}_{\mathcal{F}_{p h y s}} \otimes P_{\mathcal{H}_{e l}^{(0)}}^{\perp} P_{\Omega}^{\perp} \otimes \mathbf{1}_{\mathcal{H}_{e l}} \psi_{\alpha}),
\end{aligned}
$$

and we notice that the absolute value of (III.110) can be bound from above by $\mathcal{O}\left(\alpha^{6}\right)$, by means of a procedure similar to the treatment of (III.96). 
Returning to (III.90), we conclude that

$$
\begin{aligned}
& (I I I .72)= \\
& =(I I I .84)+(I I I .109) \\
& \quad+\mathcal{O}\left(\alpha^{5}\right)+\mathcal{O}\left(\epsilon^{\frac{1}{2}} \alpha^{\frac{9}{2}}\right)+\mathcal{O}\left(\epsilon^{-\frac{1}{4}} \alpha^{6}\right)+\mathcal{O}\left(\epsilon^{\frac{1}{8}} \alpha^{\frac{9}{2}}\right)
\end{aligned}
$$

We proceed to control the term (III.73). First, we observe that

$$
\begin{gathered}
(I I I .73)=-\alpha^{3}\left(\xi^{(0)}, \vec{A}(0)^{2} \psi_{\alpha}\right)= \\
=\quad-\alpha^{3}\left(\xi^{(0)}, \vec{A}(0)^{2} P_{\Omega} \otimes P_{\mathcal{H}_{e l}^{(1)}}^{\perp} \psi_{\alpha}\right) \\
\quad-\alpha^{3}\left(\xi^{(0)}, \vec{A}(0)^{2} P_{\Omega}^{\perp} \otimes \mathbf{1}_{\mathcal{H}_{e l}} \psi_{\alpha}\right) .
\end{gathered}
$$

The absolute value of the expression in (III.113) is trivially bounded from above by $\mathcal{O}\left(\alpha^{6}\right)$, see (III.6). As a result, we conclude that

$$
\begin{aligned}
& (\text { III.73 })= \\
& =\quad-\alpha^{3}\left(\xi^{(0)}, \vec{A}(0)^{2} P_{\Omega}^{\perp} \otimes \mathbf{1}_{\mathcal{H}_{e l}} \psi_{\alpha}\right) \\
& \quad+\mathcal{O}\left(\alpha^{6}\right) .
\end{aligned}
$$

As in the estimate of the term (III.92), we consider the following splitting of (III.116):

$$
\begin{aligned}
& -\alpha^{3}(\xi^{(0)}, \vec{A}^{(-)} \overbrace{(\alpha \vec{x}) \cdot \vec{A}^{(+)}}(\alpha \vec{x}) \mathbf{1}_{\mathcal{F}_{p h y s}} \otimes P_{\mathcal{H}_{e l}^{(0)}} P_{\Omega}^{\perp} \otimes \mathbf{1}_{\mathcal{H}_{e l}} \psi_{\alpha}) \\
& -\alpha^{3}(\xi^{(0)}, \vec{A}^{(-)} \overbrace{(\alpha \vec{x}) \cdot \vec{A}^{(+)}}(\alpha \vec{x}) \mathbf{1}_{\mathcal{F}_{p h y s}} \otimes P_{\mathcal{H}_{e l}^{(0)}}^{\perp} P_{\Omega}^{\perp} \otimes \mathbf{1}_{\mathcal{H}_{e l}} \psi_{\alpha}) \\
& -2 \alpha^{3}\left(\xi^{(0)}, \vec{A}^{(+)}(0) \cdot \vec{A}^{(-)}(0) P_{\Omega}^{\perp} \otimes \mathbf{1}_{\mathcal{H}_{e l}} \psi_{\alpha}\right) \\
& -\alpha^{3}\left(\xi^{(0)}, \vec{A}^{(-)}(0) \cdot \vec{A}^{(-)}(0) P_{\Omega}^{\perp} \otimes \mathbf{1}_{\mathcal{H}_{e l}} \psi_{\alpha}\right),
\end{aligned}
$$

by noticing that the term (III.119) vanishes, and that the terms (III.120), (III.121) can be estimated like (III.95) and (III.96), respectively. Then we can conclude that

$$
\begin{aligned}
& (I I I .73)=(I I I .118) \\
& \quad+\mathcal{O}\left(\alpha^{5}\right)+\mathcal{O}\left(\epsilon^{\frac{1}{2}} \alpha^{\frac{9}{2}}\right)+\mathcal{O}\left(\epsilon^{-\frac{1}{4}} \alpha^{6}\right)+\mathcal{O}\left(\epsilon^{\frac{1}{8}} \alpha^{\frac{9}{2}}\right)
\end{aligned}
$$

Putting it all together

Returning to the initial expression,

$$
\begin{aligned}
& \left(\xi^{(0)},\left(H_{0}-E_{\alpha}\right) \psi_{\alpha}\right)= \\
& \quad=-\left(\xi^{(0)}, H_{I} \psi_{\alpha}\right) \\
& =(I I I .72)+(I I I .73)+\mathcal{O}\left(\alpha^{5}\right)
\end{aligned}
$$


and using the equations (III.111),(III.122) we can write

$$
\begin{aligned}
& \left(\xi^{(0)},\left(H_{0}-E_{\alpha}\right) \psi_{\alpha}\right)-(I I I .118)-(I I I .109)= \\
= & (I I I .84)+\mathcal{O}\left(\alpha^{5}\right)+\mathcal{O}\left(\epsilon^{\frac{1}{2}} \alpha^{\frac{9}{2}}\right)+\mathcal{O}\left(\epsilon^{-\frac{1}{4}} \alpha^{6}\right)+\mathcal{O}\left(\epsilon^{\frac{1}{8}} \alpha^{\frac{9}{2}}\right) .
\end{aligned}
$$

We now observe that

$$
\left(\xi^{(0)},\left(H_{0}-E_{\alpha}\right) \psi_{\alpha}\right)=\left(\xi^{(0)},\left(H_{0}-E_{\alpha}\right) P_{\Omega}^{\perp} \otimes \mathbf{1}_{\mathcal{H}_{e l}} \psi_{\alpha}\right),
$$

and that we can rewrite the L.S. of (III.126) as

$$
\left(\xi^{(0)},\left(H_{0}^{\prime}-E_{\alpha}\right) P_{\Omega}^{\perp} \otimes \mathbf{1}_{\mathcal{H}_{e l}} \psi_{\alpha}\right)
$$

with

$$
\begin{aligned}
H_{0}^{\prime}:= & H_{0} \\
& -4 \alpha^{3} \mathbf{1}_{\mathcal{F}_{\text {phys }}} \otimes P_{\mathcal{H}_{e l}^{(0)}} \vec{p} \cdot \vec{A}^{(-)} \overbrace{(\alpha \vec{x}) \frac{1}{H_{0}-E_{\alpha}} \vec{p} \cdot \vec{A}^{(+)}}(\alpha \vec{x}) \mathbf{1}_{\mathcal{F}_{p h y s}} \otimes P_{\mathcal{H}_{e l}^{(0)}} \\
& +\alpha^{3} \mathbf{1}_{\mathcal{F}_{\text {phys }}} \otimes P_{\mathcal{H}_{e l}^{(0)}} \vec{A}^{(-)} \overbrace{(\alpha \vec{x}) \cdot \vec{A}^{(+)}}(\alpha \vec{x}) \mathbf{1}_{\mathcal{F}_{\text {phys }}} \otimes P_{\mathcal{H}_{\text {el }}^{(0)}} .
\end{aligned}
$$

Now, notice that

$$
\begin{aligned}
& \left(H_{0}^{\prime}-E_{\alpha}\right) \xi^{(0)}= \\
= & \iint d^{3} k d^{3} q \frac{1}{\epsilon^{\frac{1}{2}}} h_{1}\left(\frac{|\vec{k}|+|\vec{q}|+\alpha^{3} f(|\vec{k}|+|\vec{q}|)-E_{\alpha}+E^{(0)}}{\epsilon}\right) h_{2}(|\vec{k}|-|\vec{q}|) \times \\
& \times \theta_{j}(\hat{k}) \theta_{j^{\prime}}(\hat{q}) a_{j}^{*}(\vec{k}) a_{j^{\prime}}^{*}(\vec{q})\left(|\vec{k}|+|\vec{q}|+\alpha^{3} f(|\vec{k}|+|\vec{q}|)-E_{\alpha}+E^{(0)}\right) \Omega \otimes \phi^{(0)},
\end{aligned}
$$

where $f$ is defined in (III.62), with the property specified in (III.57). Hence we can estimate

$$
\begin{aligned}
& \left|\left(\xi^{(0)},\left(H_{0}^{\prime}-E_{\alpha}\right) P_{\Omega}^{\perp} \otimes \mathbf{1}_{\mathcal{H}_{e l}} \psi_{\alpha}\right)\right| \leq \\
\leq & \left\|\left(H_{0}^{\prime}-E_{\alpha}\right) \xi^{(0)}\right\|\left\|P_{\Omega}^{\perp} \otimes \mathbf{1}_{\mathcal{H}_{e l}} \psi_{\alpha}\right\| \\
\leq & \mathcal{O}\left(\epsilon \alpha^{\frac{3}{2}}\right) .
\end{aligned}
$$

Together with inequality (III.89) yields

$Q_{1}^{\prime} \epsilon^{\frac{1}{2}} \alpha^{3} \leq|(I I I .84)| \leq \mathcal{O}\left(\epsilon \alpha^{\frac{3}{2}}\right)+\mathcal{O}\left(\alpha^{5}\right)+\mathcal{O}\left(\epsilon^{\frac{1}{2}} \alpha^{\frac{9}{2}}\right)+\mathcal{O}\left(\epsilon^{-\frac{1}{4}} \alpha^{6}\right)+\mathcal{O}\left(\epsilon^{\frac{1}{8}} \alpha^{\frac{9}{2}}\right)$.

If we choose $\epsilon^{\frac{1}{2}}$ to be given by $\alpha^{\frac{3}{2}+\frac{\gamma}{2}}$, with $0<\gamma<1$, then inequality (III.135) cannot hold for $\alpha$ small enough.

Case $j=0$ 
In this case, the vector $\psi_{\alpha}$ is of the form

$$
c_{0}(\alpha) \Omega \otimes \phi^{(0)}+P_{\Omega} \otimes P_{\phi^{(0)}}^{\perp} \psi_{\alpha}+P_{\Omega}^{\perp} \otimes \mathbf{1}_{\mathcal{H}_{e l}} \psi_{\alpha},
$$

where $\phi^{(0)}$ is the unique groundstate of the atomic Hamiltonian. We know, however, (see [1]), that $\Omega \otimes \phi^{(0)}$ is asymptotic to the unique groundstate, $\psi_{g s}(\alpha)$, of the Hamiltonian $H_{\alpha}$, as $\alpha \rightarrow 0$, i.e.,

$$
\left\|\Omega \otimes \phi^{(0)}-\psi_{g s}(\alpha)\right\|=o(1) .
$$

Using (III.4), we conclude that $\psi_{\alpha}$ cannot be orthogonal to $\psi_{g s}(\alpha)$, for $\alpha$ small enough. Since $E_{\alpha}>E_{g s}$, by hypothesis, we arrive at a contradiction. This concludes our proof of Theorem III.2.

Corollary III.3. For arbitrary $\Delta_{\Sigma}>0$, there is a constant $\bar{\alpha}\left(\Delta_{\Sigma}\right)>0$ such that, for $|\alpha|<\bar{\alpha}\left(\Delta_{\Sigma}\right)$, the Hamiltonian $H_{\alpha}$ does not have any eigenvalue in the energy interval $\left(E_{g s}(\alpha), \Sigma-\Delta_{\Sigma}\right)$. 


\section{A Appendix}

The following lemma is the key ingredient to prove the bound in (III.87).

Lemma A.1. The sum of the scalar products

$$
\begin{array}{r}
\left(\phi^{(0)}, p_{j} \frac{1}{H_{e l}-E_{\alpha}+|\vec{k}|} p_{j} \phi_{l=0}^{(1)}\right) \\
+\quad\left(\phi^{(0)}, p_{j} \frac{1}{H_{e l}-E_{\alpha}+|\vec{q}|} p_{j} \phi_{l=0}^{(1)}\right)
\end{array}
$$

is not zero, for $\epsilon, \delta$, and $\alpha$ small enough, with $0<\epsilon, \alpha \ll \delta \ll 1$, where $|\vec{k}|$ and $|\vec{q}|$ fulfill the constraints

$$
E_{\alpha}-E^{(0)}-\epsilon-2 \delta \leq|\vec{k}|+\mathcal{O}\left(\alpha^{3}\right) \leq E_{\alpha}-E^{(0)}+\epsilon-\delta
$$

and

$$
\delta-2 \epsilon \leq|\vec{q}|+\mathcal{O}\left(\alpha^{3}\right) \leq 2 \delta+2 \epsilon
$$

Proof.

It suffices to show that the sum

$$
\left(\phi^{(0)}, p_{1} \frac{1}{H_{e l}-E^{(1)}+\Delta_{1}} p_{1} \phi_{l=0}^{(1)}\right)+\left(\phi^{(0)}, p_{1} \frac{1}{H_{e l}-E^{(1)}+\Delta_{2}} p_{1} \phi_{l=0}^{(1)}\right)
$$

is different from zero for $\Delta_{1}=E^{(1)}-E^{(0)}+\mathcal{O}(\delta), \Delta_{2}=\mathcal{O}(\delta)>0$, and $\delta$ small enough.

We first analyze

$$
\left(\phi^{(0)}, p_{1} \frac{1}{H_{e l}-E^{(1)}+\Delta_{1}} p_{1} \phi_{l=0}^{(1)}\right),
$$

with $\Delta_{1}=E^{(1)}-E^{(0)}+\mathcal{O}(\delta)$.

First, notice that, because of the selection rule on the orbital angular momentum of the electron, we have

$$
\begin{gathered}
\left(\phi^{(0)}, p_{1} \frac{1}{H_{e l}-E^{(1)}+\Delta} p_{1} \phi_{l=0}^{(1)}\right)= \\
=\left(\phi^{(0)}, p_{1} P_{\mathcal{H}_{e l}^{(0)}}^{\perp,+} \frac{1}{H_{e l}-E^{(1)}+\Delta_{1}} p_{1} \phi_{l=0}^{(1)}\right),
\end{gathered}
$$

therefore the expression (A.4) is well defined.

Using that

$$
p_{1}=\frac{i}{2}\left[H_{e l}, x_{1}\right]=\frac{i}{2}\left[H_{e l}-E^{(0)}, x_{1}\right],
$$


and replacing $\Delta_{1}$ by $E^{(1)}-E^{(0)}+\mathcal{O}(\delta)$, we can write

$$
\begin{aligned}
& \left(\phi^{(0)}, p_{1} \frac{1}{H_{e l}-E^{(1)}+\Delta_{1}} p_{1} \phi_{l=0}^{(1)}\right)= \\
= & -\frac{i}{2}\left(\phi^{(0)}, x_{1}\left(H_{e l}-E^{(0)}\right) \frac{1}{H_{e l}-E^{(0)}+\mathcal{O}(\delta)} p_{1} \phi_{l=0}^{(1)}\right) \\
= & -\frac{i}{2}\left(\phi^{(0)}, x_{1} p_{1} \phi_{l=0}^{(1)}\right) \\
& +\mathcal{O}(\delta) \times\left[-\frac{i}{2}\left(\phi^{(0)}, x_{1} \frac{1}{H_{e l}-E^{(0)}+\mathcal{O}(\delta)} p_{1} \phi_{l=0}^{(1)}\right)\right],
\end{aligned}
$$

where

$$
\left|\frac{1}{2}\left(\phi^{(0)}, x_{1} \frac{1}{H_{e l}-E^{(0)}+\mathcal{O}(\delta)} p_{1} \phi_{l=0}^{(1)}\right)\right|<K,
$$

with $K$ uniform in $\delta$.

Consider now

$$
\left(\phi^{(0)}, p_{1} \frac{1}{H_{e l}-E^{(1)}+\Delta_{2}} p_{1} \phi_{l=0}^{(1)}\right),
$$

with $\Delta_{2}=\mathcal{O}(\delta)>0$.

Using that

$$
p_{1}=\frac{i}{2}\left[H_{e l}, x_{1}\right]=\frac{i}{2}\left[H_{e l}-E^{(1)}, x_{1}\right],
$$

we can write

$$
\begin{aligned}
& \left(\phi^{(0)}, p_{1} \frac{1}{H_{e l}-E^{(1)}+\Delta_{2}} p_{1} \phi_{l=0}^{(1)}\right)= \\
= & \frac{i}{2}\left(\phi^{(0)}, p_{1} \frac{1}{H_{e l}-E^{(1)}+\Delta_{2}}\left(H_{e l}-E^{(1)}\right) x_{1} \phi_{l=0}^{(1)}\right) \\
= & \frac{i}{2}\left(\phi^{(0)}, p_{1} x_{1} \phi_{l=0}^{(1)}\right) \\
& -\Delta_{2} \times\left[\frac{i}{2}\left(\phi^{(0)}, p_{1} \frac{1}{H_{e l}-E^{(1)}+\Delta_{2}} x_{1} \phi_{l=0}^{(1)}\right)\right],
\end{aligned}
$$

where

$$
\begin{aligned}
& -\Delta_{2} \frac{i}{2}\left(\phi^{(0)}, p_{1} \frac{1}{H_{e l}-E^{(1)}+\Delta_{2}} x_{1} \phi_{l=0}^{(1)}\right)= \\
= & -\frac{i}{2}\left(\phi^{(0)}, p_{1} P_{\mathcal{H}_{e l}^{(1)}} x_{1} \phi_{l=0}^{(1)}\right) \\
& -\Delta_{2} \frac{i}{2}\left(\phi^{(0)}, p_{1} P_{\mathcal{H}_{e l}^{(1)}}^{\perp+} \frac{1}{H_{e l}-E^{(1)}+\Delta_{2}} x_{1} \phi_{l=0}^{(1)}\right),
\end{aligned}
$$


and

$$
\left|\frac{i}{2}\left(\phi^{(0)}, p_{1} P_{\mathcal{H}_{e l}^{(1)}}^{\perp,+} \frac{1}{H_{e l}-E^{(1)}+\Delta_{2}} x_{1} \phi_{l=0}^{(1)}\right)\right|<K,
$$

with $K$ uniform in $\delta$.

Since (A.9) and (A.15) cancel each other, we have

$$
(A .3)=-\frac{i}{2}\left(\phi^{(0)}, p_{1} P_{\mathcal{H}_{e l}^{(1)}} x_{1} \phi_{l=0}^{(1)}\right)+\mathcal{O}(\delta) .
$$

Therefore, the result to be proven follows if

$$
\left(\phi^{(0)}, p_{1} P_{\mathcal{H}_{e l}^{(1)}} x_{1} \phi_{l=0}^{(1)}\right) \neq 0 .
$$

The integrals corresponding to the scalar product in (A.22) can be calculated analytically, and the expression turns out to be different from zero as stated in Property (P2) in (I.23).

\section{References}

[1] V. Bach, J. Fröhlich, and A. Pizzo. Infrared-Finite Algorithms in QED I. The Groundstate of an Atom Interacting with the Quantized Radiation Field. Comm. Math. Phys. 2006, 264(1) 145-165.

[2] V. Bach, J. Fröhlich, and I. M. Sigal. Renormalization group analysis of spectral problems in quantum field theory. Adv. in Math. , 137:205-298, 1998.

[3] V. Bach, J. Fröhlich, and I. M. Sigal. Spectral analysis for systems of atoms and molecules coupled to the quantized radiation field. Commun. Math. Phys., 207(2):249-290, 1999.

[4] V. Bach, J. Fröhlich, I. M. Sigal, and A. Soffer. Positive commutators and the spectrum of Pauli-Fierz Hamiltonian of atoms and molecules. Commun. Math. Phys., 207(3):557-587, 1999.

[5] N.B. Delone, S.P. Goreslavsky, and V.P. Krainov. Dipole matrix elements in the quasi-classical approximation. J. Phys. B: At. Mol. Opt. Phys. 27: 4403-4419, 1994.

[6] J.Derezinski and V. Jaksic. Spectral theory of Pauli-Fierz operators. J. Func. Anal., 180(2):243-327, 2001 
[7] J.Derezinski and C.Gerard. Asymptotic completeness in quantum field theory. Massive Pauli-Fierz Hamiltonians. Rev. Math. Phys., 11(4):383450, 1999

[8] J. Fröhlich, M.Griesemer, and B. Schlein. Asymptotic completeness for Rayleigh scattering. Ann. H. Poincaré,3: 107-170, 2002

[9] J. Fröhlich, M.Griesemer, and I.M. Sigal. Mourre estimate and spectral theory for the standard model of non-relativistic QED. Mp $p_{a} r 06-316$

[10] V.Georgescu, C.Gerard, and J.S.Moller. Spectral theory of massless Pauli-Fierz models. Commun. Math. Phys., 249(1):29-78, 2004

[11] C.Gerard. A proof of the abstract limiting absorption principle by energy estimates. Mp-arc 07-43

[12] M.Griesemer. Exponential decay and ionization threshold in nonrelativistic quantum electrodynamics. J. Funct. Anal.,210 (3): 321-340, 2004

[13] M.Griesemer, E. Lieb, and M. Loss. Ground states in non-relativistic quantum electrodynamics. Invent. math.,145 (3): 557-595, 2001

[14] M.Hubner and H.Spohn. Spectral model of the spin-boson Hamiltonian. Ann. Inst. H. Poincare Phys. Theor., 62(3): 289-323, 1995

[15] A. Matsumoto. Multipole matrix elements for hydrogen atom. Physica Scripta, 44: 154-157, 1991

[16] M.Reed and B.Simon. Methods of modern mathematical physics. IV. Analysis of operators. Academic Press, New York, 1978

[17] J.Sahbani. The conjugate operator method for locally regular Hamiltonians. J. Operator Theory, 38(2): 297-322, 1997

[18] E.Skibsted. Spectral analysis of N-body systems coupled to a bosonic field. Rew. Math. Phys., 10(7): 989-1026, 1998 